\title{
Observing the metal-poor solar neighbourhood: a comparison of galactic chemical evolution predictions ${ }^{*} \dagger$
}

\author{
T. Mishenina, ${ }^{1}$ M. Pignatari, ${ }^{2,3,4}$ B. Côté, ${ }^{3,4,5,6}$ F.-K. Thielemann, ${ }^{7}$ C. Soubiran, ${ }^{8}$ \\ N. Basak, ${ }^{1}$ T. Gorbaneva, ${ }^{1}$ S. A. Korotin,,${ }^{1,9 \star}$ V. V. Kovtyukh, ${ }^{1}$ B. Wehmeyer, ${ }^{7}$ \\ S. Bisterzo, ${ }^{3,10,11}$ C. Travaglio, ${ }^{3,10,11}$ B. K. Gibson, ${ }^{2,4}$ C. Jordan, ${ }^{2,4}$
} A. Paul, ${ }^{5}$ C. Ritter $^{3,4}$ and F. Herwig ${ }^{3,4}$

${ }^{1}$ Astronomical Observatory, Odessa National University, and Isaac Newton Institute of Chile, Odessa Branch, Shevchenko Park, 650014, Odessa, Ukraine

${ }^{2}$ E.A. Milne Centre for Astrophysics, University of Hull, Hull HU6 7RX, UK

${ }^{3}$ The NuGrid Collaboration, http://www.nugridstars.org

${ }^{4}$ Joint Institute for Nuclear Astrophysics Center for the Evolution of the Elements, East Lansing, MI 48824, USA

${ }^{5}$ Department of Physics and Astronomy, University of Victoria, Victoria, BC V8W 2Y2, Canada

${ }^{6}$ National Superconducting Cyclotron Laboratory, Michigan State University, East Lansing, MI 48824, USA

${ }^{7}$ Department of Physics, University of Basel, Klingelbergstrabe 82, CH-4056 Basel, Switzerland

${ }^{8}$ Laboratoire d'Astrophysique de Bordeaux, Université Bordeaux - CNRS, B18N, allée Geoffroy Saint-Hilaire, F-33615 Pessac, France

${ }^{9}$ Crimean Astrophysical Observatory, Nauchny 298409, Crimea

${ }^{10}$ INAF, Astrophysical Observatory Turin, Strada Osservatorio 20, I-10025 Pino Torinese (Turin), Italy

${ }^{11}$ B2FH Association, I-10025 Pino Torinese, Italy

Accepted 2017 May 8. Received 2017 May 8; in original form 2016 November 27

\begin{abstract}
Atmospheric parameters and chemical compositions for 10 stars with metallicities in the region of $-2.2<[\mathrm{Fe} / \mathrm{H}]<-0.6$ were precisely determined using high-resolution, high signal-tonoise, spectra. For each star, the abundances, for 14-27 elements, were derived using both local thermodynamic equilibrium (LTE) and non-LTE (NLTE) approaches. In particular, differences by assuming LTE or NLTE are about 0.10 dex; depending on $[\mathrm{Fe} / \mathrm{H}], T_{\text {eff }}$, gravity and element lines used in the analysis. We find that the $\mathrm{O}$ abundance has the largest error, ranging from 0.10 and 0.2 dex. The best measured elements are $\mathrm{Cr}$, $\mathrm{Fe}$, and $\mathrm{Mn}$; with errors between 0.03 and 0.11 dex. The stars in our sample were included in previous different observational work. We provide a consistent data analysis. The data dispersion introduced in the literature by different techniques and assumptions used by the different authors is within the observational errors, excepting for HD103095. We compare these results with stellar observations from different data sets and a number of theoretical galactic chemical evolution (GCE) simulations. We find a large scatter in the GCE results, used to study the origin of the elements. Within this scatter as found in previous GCE simulations, we cannot reproduce the evolution of the elemental ratios $[\mathrm{Sc} / \mathrm{Fe}],[\mathrm{Ti} / \mathrm{Fe}]$, and $[\mathrm{V} / \mathrm{Fe}]$ at different metallicities. The stellar yields from core-collapse supernovae are likely primarily responsible for this discrepancy. Possible solutions and open problems are discussed.
\end{abstract}

Key words: stars: abundances - stars: late-type-Galaxy: disc-Galaxy: evolution.

\section{INTRODUCTION}

The observation of chemical abundances in stars at different metallicities provides a fundamental tool to study the evolution of our

\footnotetext{
* Based on observations collected at OHP Observatory, France.

$\dagger$ Table 7 is only available in electronic form.

^E-mail: serkor@skyline.od.ua
}

Galaxy (e.g. Reddy et al. 2003; Reddy, Lambert \& Allende Prieto 2006; Frebel 2010; Yong et al. 2013; Battistini \& Bensby 2016). Determination of parameters and chemical compositions of stars with low metal abundances is more challenging compared to stars of solar metallicity. This is due to the influence of metallicity on atmospheric parameters, caused primarily by stronger deviations from local thermodynamic equilibrium (LTE). This is associated with a decrease in electron density and reduction of collisions in reaching equilibrium (e.g. Mashonkina \& Gehren 2000). In this 
Table 1. Observation data for our target stars.

\begin{tabular}{lccc}
\hline $\mathrm{HD}$ & Date & $\mathrm{S} / \mathrm{N}$ & $\mathrm{RV}\left(\mathrm{km} \mathrm{s}^{-1}\right)$ \\
\hline 6582 & 2013 December 05 & 418 & -96.305 \\
6833 & 2006 September 19 & 234 & -243.410 \\
19445 & 2010 January 17 & 102 & -139.936 \\
22879 & 2013 December 06 & 207 & 120.397 \\
84937 & 2013 December 09 & 167 & -15.015 \\
103095 & 2013 December 06 & 259 & -97.922 \\
170153 & 2011 August 30 & 317 & 37.781 \\
216143 & 2006 September 19 & 153 & -116.462 \\
221170 & 2006 September 19 & 201 & -121.717 \\
224930 & 2011 January 16 & 326 & -41.105 \\
\hline
\end{tabular}

work, we analyse 10 stars which have been investigated by previous studies, and estimate the accuracy of determination of parameters and chemical compositions. The stars cover a metallicity range $-2.2<[\mathrm{Fe} / \mathrm{H}]<-0.6$. Stars within this range reveal crucial insights about the chemical evolution of the Galaxy. It includes the region $[\mathrm{Fe} / \mathrm{H}] \lesssim-1$, where typically only massive stars and super asymptotic giant branch (AGB) stars have sufficient time to contribute significantly to the chemical enrichment history of the Galaxy (e.g. Nomoto, Kobayashi \& Tominaga 2013). Where [Fe/H] $\gtrsim-1$, the contribution from lower mass AGB stars and supernovae type Ia (SNe Ia) affects the chemical enrichment history in the galactic disc (e.g. Matteucci \& Tornambe 1985). With this work, we aim to provide new observational data to study this metallicity region, with special attention to observational uncertainties. Most of the stars analysed have been included in previous works from other authors, and included in large stellar compilations. Relevant differences exist between different measurements for some cases. Such differences are due to legitimate assumptions and choices made by the authors. Our results are compared with a number of galactic chemical evolution (GCE) simulations. Adopting the same approach used for the observational analysis, the different simulations are discussed, where the results are a product of the theoretical setups adopted by the authors.

The paper is organized as follows: the observations and selection of stars, and definition of the main stellar parameters are described in Section 2; ages and kinematic parameters are presented in Section 3; the selection of lines is given in Section 4; the abundance determinations and the error analysis are presented in Section 5. Results, membership of galactic structures, and comparison with other data and with theoretical GCE simulations are given in Section 6. Conclusions are drawn in Section 7.

\section{OBSERVATIONS, SELECTION, AND PARAMETERS OF THE STARS}

For this study, 10 metal-poor stars with different metallicities from -0.6 to -2.2 were selected. Their spectra were obtained with the SOPHIE echelle spectrograph (Perruchot et al. 2008) attached to the $1.93 \mathrm{~m}$ telescope at the Observatoire de Haute Provence, France. The resolving power of the spectrograph is $R=75000$, the spectra are in the wavelength range $\lambda$ 4400-6800 $\AA$ and signal-to-noise ratio $(\mathrm{S} / \mathrm{N})$ of about $100-400$. The list of target stars, observation dates, S/Ns, and the radial velocity (RV) are given in Table 1.

The observations were retrieved from the online SOPHIE $\operatorname{archive}^{1}$ which provides science-ready spectra with cross-

\footnotetext{
${ }^{1}$ http://atlas.obs-hp.fr/sophie/
}

correlation functions and RV measurements. Further spectra processing (the continuum placement, equivalent width (EW) measurements, etc.) was conducted using the $\mathrm{DECH} 20$ software package by Galazutdinov (1992).

\subsection{Effective temperature $T_{\text {eff }}$}

The main methods to calculate $T_{\text {eff }}$ are based on photometric calibrations and on spectroscopic calibrations using Fe abundance lines; assuming the absence of any relationship between the elemental abundance estimated by a certain spectral line and the lower excitation potential $E_{\text {low }}$ of the line for a given temperature. In this study, we applied the colour- $T_{\text {eff }}$ calibrations of the $B-V$ and $b-y$ colour indices for dwarfs (Alonso, Arribas \& Martinez-Roger 1996) and giants (Alonso, Arribas \& Martínez-Roger 1999), taking into account the stellar metallicity.

The $B-V$ and $b-y$ data were taken from the SIMBAD data base. The $T_{\text {eff }}$ determinations for different values of the $B-V$ and $b-y$ colour indices for our stars, and the $T_{\text {eff }}$ values obtained using spectroscopic methods are presented in Table 2. Fig. 1 shows the dependence of the iron abundance $\log \mathrm{A}(\mathrm{Fe} \mathrm{I})$ on the lower excitation potential $E_{\text {low }}$ for each target star, where an abundance of the hydrogen is $\log \mathrm{A}(\mathrm{H})=12.0$.

By using different colour indices $(B-V$ or $b-y)$, there is an average variation of $T_{\text {eff }}$ of $50 \mathrm{~K}$, with the maximum difference never exceeding $100 \mathrm{~K}$ (see Table 2). The comparison of these results with the spectroscopic $T_{\text {eff }}$ gives higher discrepancies $\left(\Delta T_{\text {eff }}>100 \mathrm{~K}\right)$, particularly when using the $B-V$ colour index. We find the opposite situation for star HD6833, as the $b-y$ colour index of $T_{\text {eff }}$ determination results in higher difference between the $T_{\text {eff }}$ values. However, for the star HD224930 both colour indices give $\Delta T_{\text {eff }}>100 \mathrm{~K}$.

It is important to account for the reddening of $E(B-V)$ in $T_{\text {eff }}$, and other parameter determinations based on photometric calibrations. Our investigated stars are in close vicinity to the Sun meaning most of them have little reddening. Accounting for the reddening for more distant stars, like the star HD221170 in our sample, can

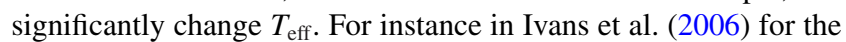
star HD221170, it was shown that usage of larger $E(B-V)$ value of reddening resulted in increased temperature $(4610 \mathrm{~K})$. At this temperature, the authors observe the dependence of the iron abundance on the lower excitation potential $E_{\text {low }}$ for a given line, which should not be if the $T_{\text {eff }}$ is correctly defined. Given these uncertainties, we opted to use the spectroscopic method for the temperature determination (see Fig. 1). We are aware that the accuracy of this method depends on the oscillator strengths used in the calculations with allowance of deviations from LTE [non-LTE (NLTE)]. As reported in the studies by Mashonkina et al. (2011) and Sitnova et al. (2015) for the stars with $[\mathrm{Fe} / \mathrm{H}]>-1$ and effective temperatures up to $5800 \mathrm{~K}$, the NLTE corrections for neutral iron are smaller than 0.05 dex and they increase with decreasing metallicity. This study also shows deviations from LTE for iron do not exceed 0.05 dex for our target stars with $[\mathrm{Fe} / \mathrm{H}]$ of about -2.25 dex and $T_{\text {eff }}<5000 \mathrm{~K}$. Only for the stars HD84937 and HD19445 do these deviations reach of order 0.05-0.12 dex; depending on the excitation potential of the employed iron lines the deviations from LTE decrease with increasing excitation potential (Bergemann et al. 2012). Most neutral iron lines used in our calculations have the lower excitation potential more than $2 \mathrm{eV}$. However, the estimated effect of deviations from LTE on the neutral iron lines varies significantly across different studies. This is due to the complexity of the multilevel model of an iron atom, which requires a large amount of atomic data 
Table 2. Parameters of studied stars.

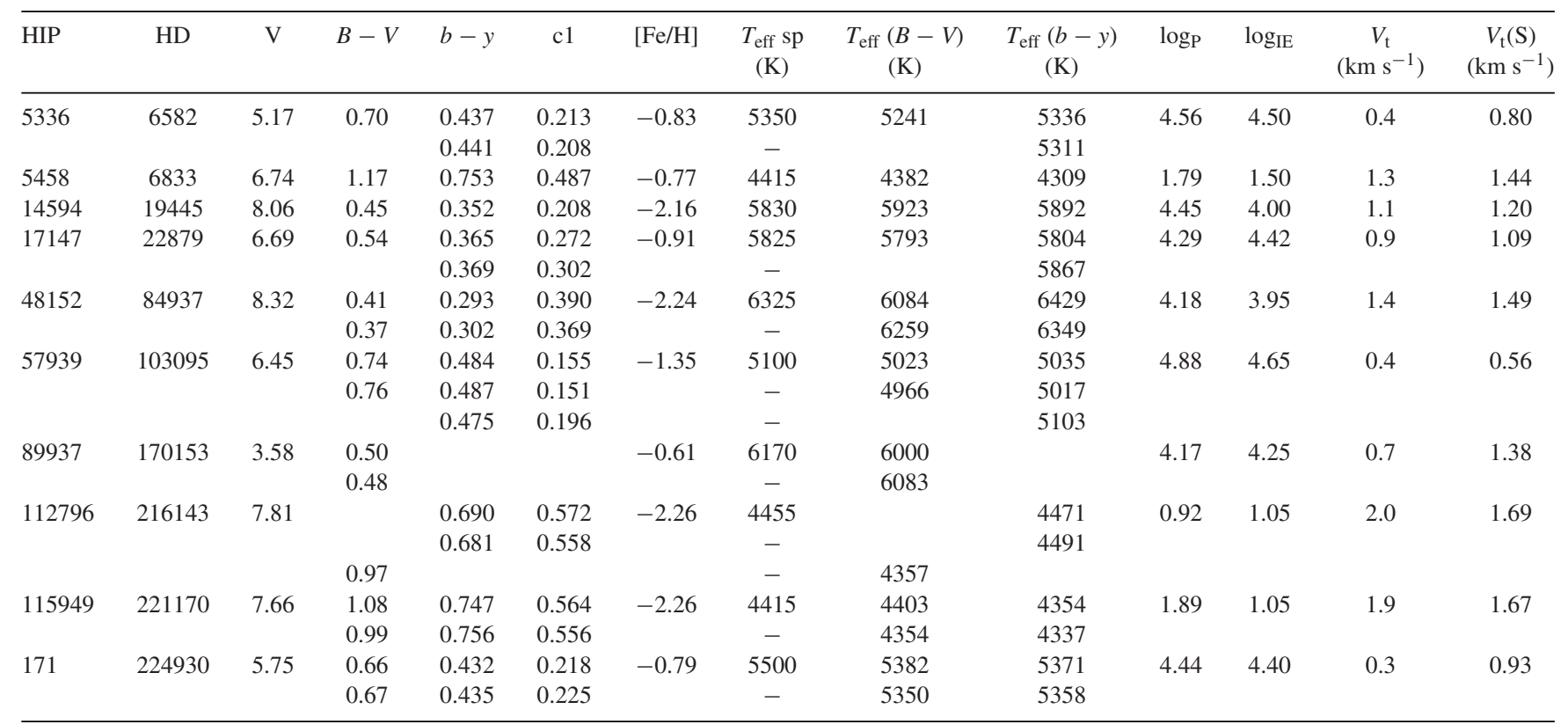

Notes: The values of $V_{\mathrm{t}}(\mathrm{S})$ are calculated by using the parametric formula by Sitnova et al. (2015).

(for which there are high uncertainties). That is one of the arguments in favour of application of the LTE analysis. Additionally, the majority of metal-poor stars' chemical composition estimates, and their in-depth study, have been performed under the assumption of LTE for parameter and metallicity determinations.

\subsection{Surface gravities $\log g$}

For the target stars, we used two methods for determination of log $g$ : (1) standard formula using the parallax $(P)$ :

$\log g_{\mathrm{P}}=-12.50+\log \left(\mathrm{M} / \mathrm{M}_{\odot}\right)+4 T_{\text {eff }}+0.4(M v+\mathrm{BC})$,

where $\mathrm{M} / \mathrm{M}_{\odot}-$ mass of the star in units of solar masses, Mv - absolute magnitude, BC - bolometric correction; bolometric corrections are taken from Flower (1975), absolute magnitude is determined by the parallax $P$ from the catalogue Hipparcos (van Leeuwen 2007); and (2) iron ionization equilibrium (IE, spectroscopic method) of the neutral and ionized iron. This method implies that similar abundances are obtained from the neutral iron $\mathrm{Fe}_{\mathrm{I}}$ and ionized iron $\mathrm{Fe}$ II lines.

Table 2 presents the $\log g$ determinations by the two methods, namely $\log g_{\mathrm{P}}$ and $\log g_{\mathrm{IE}}$, respectively. We obtained a good agreement between the $\log g$ definitions with these two methods, except for two stars, namely HD19445 and HD221170. In our opinion, this is due to taking into account the reddening at the gravity definitions using parallax. For instance, accounting for the error in the parallax determination for HD221170 resulted in $\log g$ from 1.90 to 1.48 , while factoring in the $E(B-V)$ reduced the value of $\log g$ down to 1.66 with the parallax $P=0.00294$. When determining $\log g$ using the parallax $\log g_{\mathrm{P}}$, the primary error is introduced by the accuracy of the parallax itself and by the accounting for the reddening; when using spectroscopic method for determination of $\log g\left(\log g_{\mathrm{IE}}\right)$, the use of oscillator strengths and accounting for deviations from LTE are essential. However, as shown in Bergemann et al. (2012), a small NLTE correction is needed to establish IE at solar metallicities, while for very metal-poor stars these effects reach only of +0.1 dex on Fe I lines. Fe II lines are basically not affected by departures from LTE. Since the effect of NLTE on $\log g$ determination is rather small (see also Jofré et al. 2014), in this study we used the spectroscopic determinations of $\log g$.

\subsection{Turbulent velocity $V_{\mathrm{t}}$}

The turbulent velocity $V_{\mathrm{t}}$ was defined by assuming the absence of correlation between the $\mathrm{Fe}$ abundance, estimated by the $\mathrm{Fe}_{\mathrm{I}}$ line, and its EW (Fig. 1). In Table 2, we compare our $V_{\mathrm{t}}$ determinations with the calculations by Sitnova et al. (2015), obtained by using the parametric formula calibrated over a large number of stars:

$V_{\mathrm{t}}=-0.21+0.06[\mathrm{Fe} / \mathrm{H}]+5.6\left(T_{\text {eff }} / 10^{4}\right)-0.43 \log g$

The $V_{\mathrm{t}}$ determinations obtained by this formula (Sitnova et al. 2015) are in good agreement with our determinations, with the exceptions of: HD6582, 103095, and 224930. For these stars, our determinations are lower by $0.3-0.5$ dex. The star HD224930 is also included in Takeda (2007), where they report a $V_{\mathrm{t}}=0.1 \mathrm{~km} \mathrm{~s}^{-1}$, that is 0.2 dex lower than our estimation and 0.8 dex lower than Sitnova's formula (Sitnova et al. 2015).

The adopted value of the metallicity $[\mathrm{Fe} / \mathrm{H}]$ was calculated using the $\mathrm{Fe}$ abundance obtained from the Fe I lines.

\subsection{Comparison of our parameter values with other authors, and error determinations}

In this section, we compare our atmospheric parameters to those obtained by other authors and we estimate the impact of deviations from LTE on the parameter determination.

The stars HD6582, HD22879, HD84937, and HD103095 are Gaia benchmark stars which have $T_{\text {eff }}$ and $\log g$ determined from fundamental relations, independently of spectroscopy (Heiter et al. 2015). These values and ours are compared in Table 3.

We see agreement between our data and those by Heiter et al. (2015) within the stated error definitions, except for the star HD103095 (Gmb 1830). However, there is no agreement between our and Heiter et al. (2015) results, considering $2 \sigma$ errors. A detailed discussion that is useful to explain the temperature discrepancy for this star was presented in Heiter et al. (2015). The authors preferred 

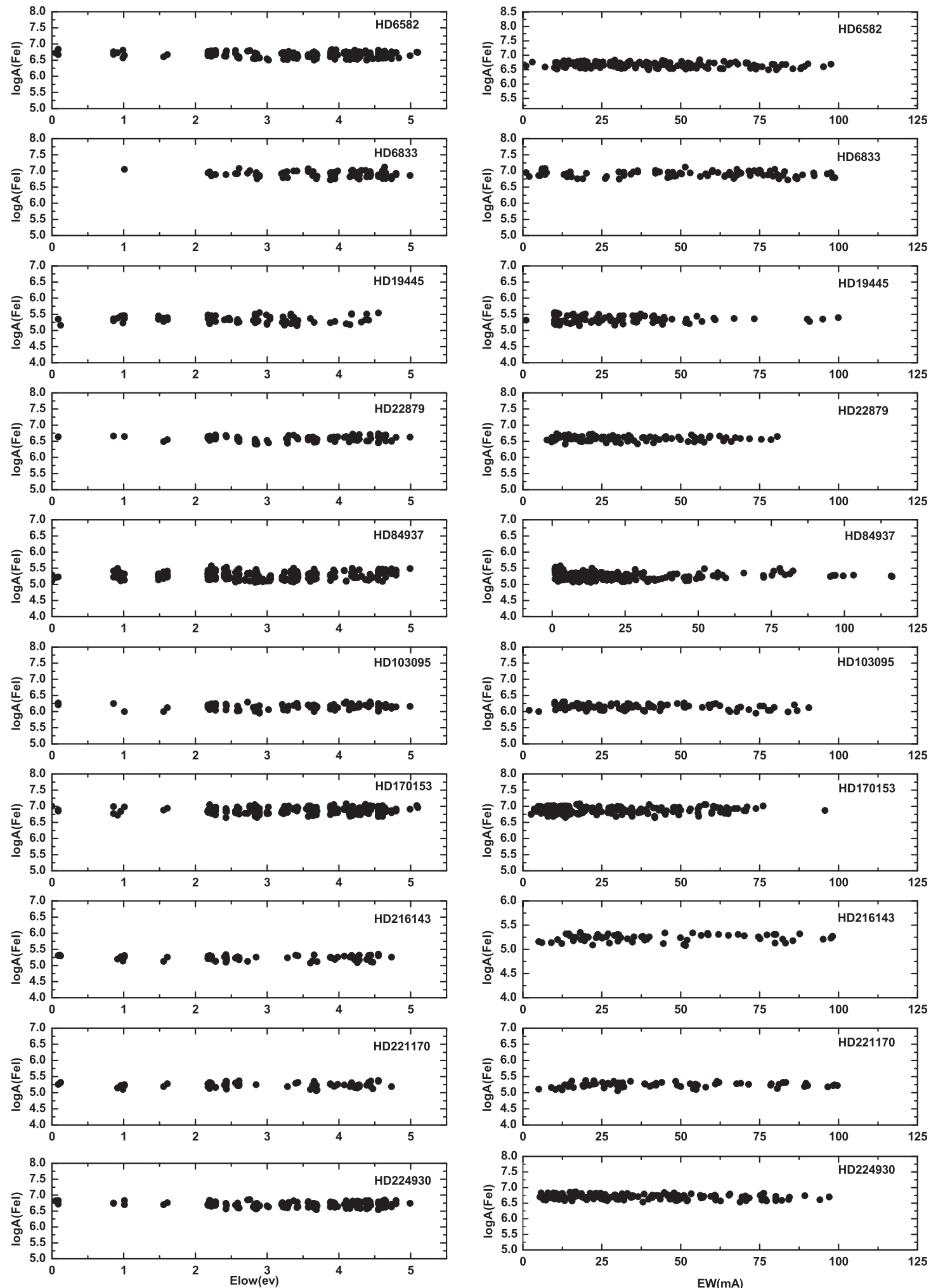

Figure 1. $(\mathrm{Fe} / \mathrm{H})$ versus $E_{\text {low }}$ and $\mathrm{EW}$.

the value $T_{\text {eff }}$ obtained by the method, based on measuring the diameter of star. However, they summed up that the further interferometric observations at longer baselines and/or shorter wavelengths are clearly needed to resolve or confirm the $T_{\text {eff }}$ discrepancy for Gmb 1830. The temperature values obtained for this star in other works are $5168 \mathrm{~K}$ (Casagrande et al. 2011) and 5129 K (González
Hernández \& Bonifacio 2009), are much closer to the value that we used.

The parameter determinations by other authors in the last $15 \mathrm{yr}$ are presented in Table A2 for the same stars.

HD6582. The average values of the parameters obtained in different studies are $T_{\text {eff }}=5336 \mathrm{~K} ; \log g=4.44$; and $[\mathrm{Fe} / \mathrm{H}]=-0.86$, 
Table 3. Parameters of our target stars and comparison with Heiter et al. (2015) for four common Gaia benchmark stars.

\begin{tabular}{lccccc|cccc}
\hline & \multicolumn{3}{c}{ Heiter et al. (2015) } & \multicolumn{3}{c}{ Our } \\
HD (name) & $T_{\text {eff }}(\mathrm{K})$ & $\sigma, \pm$ & $\log g$ & $\sigma, \pm$ & & $T_{\text {eff }}(\mathrm{K})$ & $\log g$ & $\Delta T_{\text {eff }}(\mathrm{K})$ & $\Delta \log g$ \\
\hline $6582(\mu$ Cas) & 5308 & 29 & {$[4.41]$} & {$[0.06]$} & $\mid$ & 5350 & 4.5 & 42 \\
22879 & 5868 & 89 & 4.27 & 0.04 & $\mid$ & 5825 & 4.42 & -43 \\
84937 & 6356 & 97 & 4.06 & 0.04 & $\mid$ & 6325 & 3.95 & -31 & 0.15 \\
103095 (Gmb 1830) & {$[4827]$} & {$[55]$} & 4.60 & 0.03 & $\mid$ & 5100 & 4.65 & 273 \\
\hline
\end{tabular}

Note: The values between square brackets are not obtained directly, therefore we consider a $2 \sigma$ error.

and they agree with our determinations within $1 \sigma$. The star ( $\mu$ Cas) is one of Gaia FGK benchmark stars (Jofré et al. 2014).

HD6833. The mean parameters for this star obtained in different studies are $T_{\text {eff }}=4425 \mathrm{~K} ; \log g=1.32 ;$ and $[\mathrm{Fe} / \mathrm{H}]=-0.95$, and they agree with our determinations. This star has a peculiar chemical composition, and belongs to the $\mathrm{CN}$-weak giants (Luck 1991).

HD19445. This is a well-known benchmark star for many studies. The mean parameters for this star are $T_{\text {eff }}=5973 \mathrm{~K} ; \log g=4.34$; and $[\mathrm{Fe} / \mathrm{H}]=-2.03$. Our $T_{\text {eff }}$ determinations differ from the mean value by $143 \mathrm{~K}$. This is the only star for which the difference exceeds our assumed accuracy $( \pm 100 \mathrm{~K})$, but they are still consistent within $1 \sigma$. High $T_{\text {eff }}$ values are also reported by Casagrande et al. (2010) and VandenBerg et al. (2014). In the study by Casagrande et al. (2010), $T_{\text {eff }}$ was determined using the method based on the infrared fluxes. The same $T_{\text {eff }}$ determination was adopted by VandenBerg et al. (2014).

HD22879. This star is well studied, used for different comparison of stellar parameters and chemical composition (Jofré et al. 2014; Sitnova et al. 2015). Our determinations are consistent with the mean parameter values, which are $T_{\text {eff }}=5853 \mathrm{~K}$; $\log g=4.37$; and $[\mathrm{Fe} / \mathrm{H}]=-0.83$. For this star, our stellar parameters under LTE approximation are in good concordance with the NLTE determinations reported in Sitnova et al. (2015) within the limits of the stated accuracy. This is due to the fact that the difference in the determinations for the line $\left(\mathrm{Fe}_{\mathrm{I}}-\mathrm{Fe}\right.$ II) in LTE is $-0.06 \pm 0.08$, and it is very close to the determination made under NLTE approximation which equals $-0.03 \pm 0.08$.

HD84937. This star was analysed several times by previous work (e.g. Jofré et al. 2014; Sitnova et al. 2015). Our determinations agree with the mean parameters for this star, $T_{\text {eff }}=6353 \mathrm{~K}$; $\log g=4.04$; and $[\mathrm{Fe} / \mathrm{H}]=-2.09$. In Bensby, Feltzing \& Oey (2014), the given $T_{\mathrm{eff}}$ is much higher compared to other authors. Our results under LTE approximation also agree with the NLTE determinations by Sitnova et al. (2015), within the stated accuracy. However, the difference in the LTE and NLTE determinations for the ( $\mathrm{Fe}_{\mathrm{I}}-\mathrm{Fe}$ II) lines for this star is slightly higher than for HD22879: $-0.06 \pm 0.11$ and $0.0 \pm$ 0.12 , respectively.

HD103095. This star (Gmb 1830) is one of the Gaia benchmark stars (Jofré et al. 2014). Our mean parameters are $T_{\text {eff }}=5066 \mathrm{~K}$, $\log g=4.61$, and $[\mathrm{Fe} / \mathrm{H}]=-1.29$. They agree with the determinations under NLTE approximation by Sitnova et al. (2015). The difference in the determinations under LTE and NLTE approximation is close to zero.

HD170153. Our parameters determinations are in good agreement with the mean values $T_{\text {eff }}=6104 \mathrm{~K}, \log g=4.25$, and $[\mathrm{Fe} / \mathrm{H}]$ $=-0.62$.

$H D 216143$. The differences between our results and the mean parameter values $T_{\text {eff }}=4526 \mathrm{~K}, \log g=1.02$, and $[\mathrm{Fe} / \mathrm{H}]=-2.20$ are within the given errors.

HD221170. The mean parameters are $T_{\text {eff }}=4481 \mathrm{~K}, \log$ $g=0.97$, and $[\mathrm{Fe} / \mathrm{H}]=-2.12$, in agreements with our results.
Table 4. The comparison of our parameter determinations with those of other authors: mean differences and rms deviations, and $n$ - number of common stars.

\begin{tabular}{lcccc}
\hline$<\Delta T_{\text {eff }}>(\mathrm{K})$ & $<\Delta \log g>$ & $<\Delta[\mathrm{Fe} / \mathrm{H}]>$ & $n$ & References \\
\hline $60 \pm 166$ & $0.04 \pm 0.11$ & - & 4 & Heiter et al. (2015) \\
$-11 \pm 46$ & $0.04 \pm 0.14$ & - & 3 & Heiter et al. (2015) \\
$22 \pm 96$ & $-0.05 \pm 0.20$ & $-0.09 \pm 0.08$ & 6 & Gratton et al. (2003) \\
$29 \pm 107$ & $0.07 \pm 0.15$ & $0.05 \pm 0.14$ & 9 & Fulbright (2000) \\
$-16 \pm 66$ & $0.00 \pm 0.14$ & $-0.04 \pm 0.10$ & 10 & Mean values \\
\hline
\end{tabular}

The star is one of the most well-known $r$-process stars, used as a benchmark for $r$-process nucleosynthesis in the early Galaxy and in comparison with the $r$-process abundances in the Solar system (e.g. Fulbright 2000; Burris et al. 2000; Ivans et al. 2006; MolendaŻakowicz et al. 2013).

HD224930. Our determinations are in good agreement with the mean parameters $T_{\text {eff }}=5429 \mathrm{~K}, \log g=4.36$, and $[\mathrm{Fe} / \mathrm{H}]=-0.78$.

The discrepancies between our results and the average values for 10 stars in our sample are given by:

$<\left(T_{\text {effour }}-<T_{\text {eff star }}>\right)>=<\Delta T_{\text {eff }}>, \mathrm{K}$,

$<\left(\log g_{\text {our }}-<\log g_{\text {star }}>\right)>=<\Delta \log g>$,

$<\left([\mathrm{Fe} / \mathrm{H}]_{\text {our }}-<[\mathrm{Fe} / \mathrm{H}]_{\text {star }}>\right)>=<\Delta[\mathrm{Fe} / \mathrm{H}]>$

and are presented in Table 4 . The $\Delta$ values are the mean difference of our values minus the average values obtained in other studies.

Summing up, the stellar parameters derived in this work are in good agreement with the results obtained in the literature. Based on Table 4, we derive as errors for the effective temperature $\Delta T_{\text {eff }}=$ $\pm 100 \mathrm{~K}$, for the surface gravity $\Delta \log g= \pm 0.2$, and for the microturbulent velocity $\Delta V_{\mathrm{t}}= \pm 0.1$.

\section{AGES AND KINEMATIC PARAMETERS}

In Holmberg, Nordström \& Andersen (2009), Takeda (2007), Maldonado et al. (2012), VandenBerg et al. (2014), Delgado Mena et al. (2014), Bensby et al. (2014), Ramírez et al. (2012), and Ramírez, Allende Prieto \& Lambert (2013), the stellar ages were determined for seven stars included in our sample (see Table 5). The age spread does not usually exceed 2 Gyr. This is consistent with the stated accuracy across all studies except Holmberg et al. (2009). In their work, for HD19445 and HD22849, the authors provide a different value for $T_{\text {eff }}$ for age determination. A detailed study of the stellar ages for the stars HD19445 and HD84937 is presented by VandenBerg et al. (2014), using both evolution tracks and the Wilkinson Microwave Anisotropy Probe (WMAP) observations. In the study by Ivans et al. (2006), the HD221170 star age was estimated using the $\mathrm{Th} / \mathrm{Eu}$ ratio and equals $11.7 \pm 2.8 \mathrm{Gyr}$. This result is consistent with cosmic age determinations by the WMAP experiment [14.1 Gyr, Tegmark et al. (2004), and 13.7 Gyr, Spergel et al. (2003)], with determinations of the main-sequence turnoff ages for 
Table 5. Ages of our target stars and comparison with data of other authors.

\begin{tabular}{|c|c|c|c|c|c|c|c|c|c|c|c|c|}
\hline HD & $\begin{array}{c}\text { Age (Gyr) } \\
\text { Bressan (2012) }\end{array}$ & Girardi (2002) & 1 & 2 & 3 & 4 & 5 & 6 & 7 & 8 & 9 & 10 \\
\hline 6582 & 11.10 & 9.31 & - & 10.19 & - & - & - & - & - & - & 2.10 & 1.90 \\
\hline 6833 & 8.96 & 9.61 & - & - & - & - & - & - & - & - & & \\
\hline 22879 & 11.48 & 11.74 & 7.5 & - & - & - & - & - & 12.85 & 13.02 & 13.80 & 13.30 \\
\hline 84937 & 11.46 & 11.47 & - & - & - & 12.09 & 11.38 & 10.2 & - & - & & \\
\hline 103095 & 7.08 & 5.26 & - & 10.19 & - & - & - & - & - & 13.87 & & \\
\hline 221170 & 9.24 & 9.55 & - & - & - & - & - & - & - & - & & \\
\hline 224930 & 10.83 & 10.14 & 14.7 & 10.19 & 12.7 & - & - & - & 6.3 & 14.46 & & \\
\hline
\end{tabular}

Notes: 1 - Holmberg et al. (2009), 2 - Takeda (2007), 3 - Maldonado et al. (2012), 4 - VandenBerg et al. (2014), 5 - Delgado Mena et al. (2014), 6 - Bensby et al. (2014), 7 - Ramírez et al. (2012), 8 - Ramírez et al. (2013), 9 and 10 - correspond to Padova and BASRI isochrones, see in detail in Casagrande et al. (2011).

Table 6. Kinematical parameters of studied stars.

\begin{tabular}{|c|c|c|c|c|c|c|c|c|c|c|c|c|c|c|}
\hline HD & $\begin{array}{l}\text { Dist } \\
\mathrm{pc}\end{array}$ & $\begin{array}{l}\text { Ref } \\
(\mathrm{P})\end{array}$ & $\begin{array}{c}\operatorname{err}_{\pi} \\
\text { per cent }\end{array}$ & $\begin{array}{c}U_{v} \\
\mathrm{~km} \mathrm{~s}^{-1}\end{array}$ & $\begin{array}{c}V_{v} \\
\mathrm{~km} \mathrm{~s}^{-1}\end{array}$ & $\begin{array}{c}W_{v} \\
\mathrm{~km} \mathrm{~s}^{-1}\end{array}$ & ecc & $\begin{array}{c}\text { Rmin } \\
\mathrm{kpc}\end{array}$ & $\begin{array}{c}\mathrm{Rmax} \\
\mathrm{kpc}\end{array}$ & $\begin{array}{c}\text { Rmean } \\
\text { kpc }\end{array}$ & $\begin{array}{c}\text { Zmin } \\
\mathrm{kpc}\end{array}$ & $\begin{array}{c}\mathrm{Zmax} \\
\mathrm{kpc}\end{array}$ & $\begin{array}{c}\text { Zmean } \\
\mathrm{kpc}\end{array}$ & Pop \\
\hline 6582 & 8 & $\mathrm{H}$ & 0.5 & -44 & -156 & -36 & 0.71 & 1.5 & 8.6 & 6.01 & -0.4 & 0.4 & 0.2 & halo or thick \\
\hline 6833 & 197 & $\mathrm{~T}$ & 10.0 & 123 & -202 & 68 & 0.93 & 0.4 & 10.9 & 7.1 & -6.5 & 6.6 & 1.9 & accreted halo \\
\hline 19445 & 39 & $\mathrm{~T}$ & 0.9 & 157 & -123 & -68 & 0.69 & 2.3 & 12.2 & 8.75 & -1.5 & 1.5 & 0.8 & halo \\
\hline 22879 & 26 & $\mathrm{~T}$ & 0.8 & -120 & -81 & -38 & 0.47 & 3.7 & 10.4 & 7.7 & -0.5 & 0.5 & 0.3 & halo or thick \\
\hline 84937 & 80 & $\mathrm{H}$ & 8.5 & 226 & -238 & -7 & 0.98 & 0.2 & 15.4 & 10.6 & -9.2 & 9.1 & 1.6 & halo \\
\hline 103095 & 9 & $\mathrm{H}$ & 0.7 & 281 & -158 & -13 & 0.91 & 1.1 & 22.1 & 15.6 & -0.2 & 0.2 & 0.1 & halo \\
\hline 221170 & 448 & $\mathrm{~T}$ & 14.8 & 105 & -132 & -23 & 0.63 & 2.3 & 10.0 & 7.2 & -0.3 & 0.4 & 0.2 & halo \\
\hline 224930 & 12 & $\mathrm{H}$ & 3.8 & -7 & -76 & -29 & 0.32 & 4.4 & 8.5 & 6.7 & -0.3 & 0.3 & 0.2 & thick disc \\
\hline
\end{tabular}

Notes: Dist - distance; Ref - parallax P sources: H - (van Leeuwen 2007), T - (Gaia Collaboration et al. 2016); $\operatorname{err}_{\pi}$ - parallax determination error; $U_{v}, V_{v}, W_{v}$ - components of heliocentric space velocity; ecc - eccentricity; Rmin, Rmax, Rmean - galactic distances; Zmin, Zmax, Zmean - distances from the galactic plane; Pop - belonging to the type of the galaxy population.

globular clusters [14 Gyr, Cho et al. (2016)] and with the results by Planck (13.80 \pm 0.04 Gyr, Planck Collaboration I 2016).

In this work, we defined the age using the tool available online at http://stev.oapd.inaf.it/cgi-bin/param, using the stellar tracks by Bressan et al. (2012) and Girardi et al. (2002). For our stars with $[\mathrm{Fe} / \mathrm{H}]<-2.10$, we used as input parameter the same value of $[\mathrm{Fe} / \mathrm{H}]=-2.10$ for each from these stars because the tracks limited by $[\mathrm{Fe} / \mathrm{H}]=-2.20$. The results of age determinations are presented in Table 5. For stars HD103095 and 216143, we found the differences that are more than \pm 2 Gyr. However, the errors are large enough to allow for this: for HD103095 those are 7.075 $\pm 3.930 \mathrm{Gyr}$ (Bressan et al. 2012) and 5.261 \pm 4.089 Gyr (Girardi et al. 2002), and for HD216143 those are 6.516 \pm 4.441 Gyr (Bressan et al. 2012) and $2.906 \pm 2.938$ Gyr (Girardi et al. 2002). So, we find an agreement with the data of other authors, within the determination accuracy.

Kinematic and spatial characteristics of our target stars are important features in terms of their location in the Galaxy and dynamic evolution of the Galaxy. Distances and heliocentric velocities have been derived with the parallaxes and proper motions from the newly released the Tycho-Gaia Astrometric Solution catalogues (Gaia Collaboration et al. 2016) or from Hipparcos (van Leeuwen 2007), combined to the RVs of Table 1 . The orbital parameters have been computed by integrating the equations of motion in the galactic model of Allen \& Santillan (1993), adopting a default value of $10 \mathrm{Gyr}$ as the integration time. The adopted velocity of the Sun with respect to the local standard of rest (LSR) is $(9.7,5.2,6.7) \mathrm{km} \mathrm{s}^{-1}$
(Bienaymé 1999), the solar galactocentric distance $R=8.5 \mathrm{kpc}$, and circular velocity $\mathrm{V}_{\mathrm{LSR}}=220 \mathrm{~km} \mathrm{~s}^{-1}$. The kinematical parameters are reported in Table 6 .

\section{SELECTION OF LINES}

Since the stars in our sample cover a large range of temperatures ( $4400 \mathrm{~K}<T_{\text {eff }}<6300 \mathrm{~K}$, including F-, G-, K-type dwarfs and giants) and metallicities $(-2.26<[\mathrm{Fe} / \mathrm{H}]<-0.6)$, it is necessary to create a list (or lists) of unblended lines for different parameter ranges to determine the chemical composition of the stars. To create the list for iron and $s$-and $r$-process capture lines, we adopted data from several studies, including Sneden et al. (2009), Lawler et al. (2006, 2009), Takeda et al. (2005), Lai et al. (2008), Aoki et al. (2007), Ramírez, Allende Prieto \& Lambert (2007), Simmerer et al. (2004), Den Hartog et al. (2006), Colucci et al. (2012), Bensby et al. (2014), and Roederer et al. (2014a). We selected about four thousand lines of different chemical elements. The atomic parameters of the list of lines were taken from the VALD data base (Kupka et al. 1999). The Fe I, Fe II, Ti I, Ni I, Cr I, Co I, and V I lines were selected using the high-resolution solar spectrum obtained with the same spectrograph SOPHIE. Amongst more than 1000 initially selected lines, we choose from 102 (HD84937) to 303 (HD6582) unblended lines, which are optimal for the measurements at different metallicities. Nine of the Mn I (4502, 4709, 4761, 4762, 4783, 4823, 5432, 6013, and $6021 \AA \AA$ A ) lines were used to determine the manganese abundance; this list of lines was reported in the study by Prochaska \& 
Table 7. Atomic data and EWs of used lines.

\begin{tabular}{lccccc}
\hline HD & $\begin{array}{c}\lambda, \\
\AA\end{array}$ & El & $\begin{array}{c}\text { EW } \\
(\mathrm{m} \AA)^{\sim}\end{array}$ & $\begin{array}{c}\log g f \\
(\mathrm{eV})\end{array}$ & $\mathrm{E}_{\text {low }}$ \\
\hline 6582 & 5517.533 & $\mathrm{Si}$ & 6.7 & -2.609 & 5.082 \\
6582 & 5645.613 & $\mathrm{Si}$ I & 16.2 & -2.139 & 4.930 \\
6582 & 5665.554 & $\mathrm{Si}$ & 18.1 & -2.039 & 4.920 \\
6582 & 5684.484 & $\mathrm{Si}$ I & 33.0 & -1.649 & 4.954 \\
- & - & - & - & - & - \\
\hline
\end{tabular}

Notes: Table 7 is only available in electronic form.

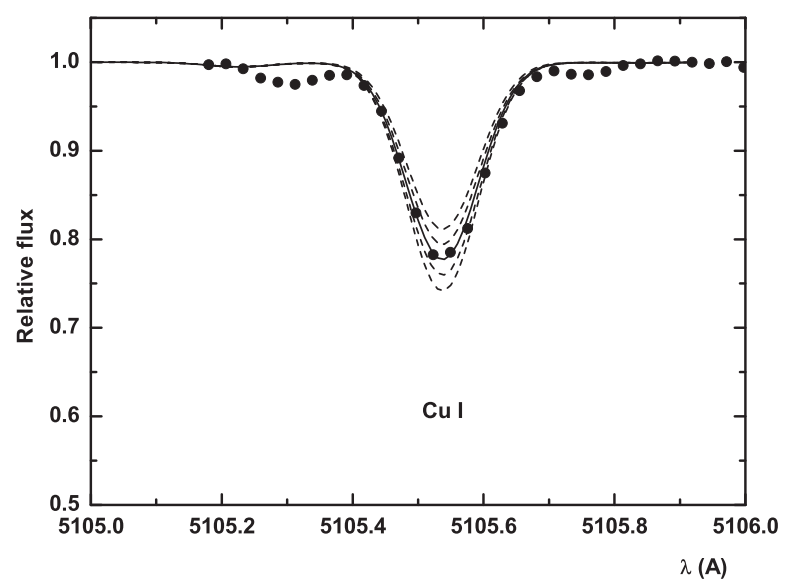

Figure 2. Observed (dots) and calculated (solid and dashed lines) spectra in the region of $\mathrm{Cu}$ I line for HD22879, the change in the $\mathrm{Cu}$ abundance is 0.05 dex.

McWilliam (Prochaska \& McWilliam 2000). The abundances of neutron-capture elements (Y, Zr, $\mathrm{La}, \mathrm{Pr}, \mathrm{Nd}, \mathrm{Sm}, \mathrm{Eu}$, and $\mathrm{Gd}$ ) were determined by the lines Y II (4-10 lines), Zr II (2-10 lines), La II (4-9 lines), Pr II (1-5 lines), Nd II (5-11 lines), Sm II (9-11 lines), Eu II (1-2 lines), and Gd II (3-5 lines). The list of the lines with their atomic parameters and EWs in the spectra of all stars is presented in Table 7, the atomic data for the lines used in synthetic method calculations are presented in Table A1.

\section{DETERMINATION OF CHEMICAL COMPOSITIONS (ELEMENTAL ABUNDANCES)}

The abundances of the investigated elements: $\mathrm{Li}, \mathrm{O}, \mathrm{Na}, \mathrm{Mg}, \mathrm{Al}$, $\mathrm{Si}, \mathrm{Ca}, \mathrm{Ni}, \mathrm{Co}, \mathrm{Mn}, \mathrm{Y}, \mathrm{Zr}, \mathrm{Ba}, \mathrm{La}, \mathrm{Ce}, \mathrm{Nd}, \mathrm{Sm}, \mathrm{Eu}$, and $\mathrm{Gd}$ were determined for our target stars under LTE and NLTE approximations using the atmosphere models by Castelli \& Kurucz (2004). The Fe, Ti, V, Cr, Ni, and Co abundances were determined using the EWs and the WIDTH9 code by Kurucz (1993). The Mn, Y, Zr, La, Pr, $\mathrm{Ce}, \mathrm{Nd}, \mathrm{Sm}, \mathrm{Eu}$, and $\mathrm{Gd}$ abundances were determined using a new version of the STARSP synthetic spectrum code (Tsymbal 1996). The oscillator strengths for these lines were adopted from the VALD data base (Kupka et al. 1999). The Mn, Eu, and Pr abundances were estimated accounting for the hyperfine structure: for the Mn I lines, the hyperfine splitting (HFS) data were taken from Prochaska \& McWilliam (2000). The van der Waals damping constant C6 was adopted from Bergemann \& Gehren (2008). The HFS data for the Eu II lines (4129 and 6645 A) were adopted from Ivans et al. (2006), and for the Pr II lines from Sneden et al. (2009). The La and Sm lines were so weak that the HFS can be neglected. The spectrum synthesis fitting of the $\mathrm{Cu}$ and $\mathrm{Y}$ lines to the observed profiles is shown in Figs 2 and 3.

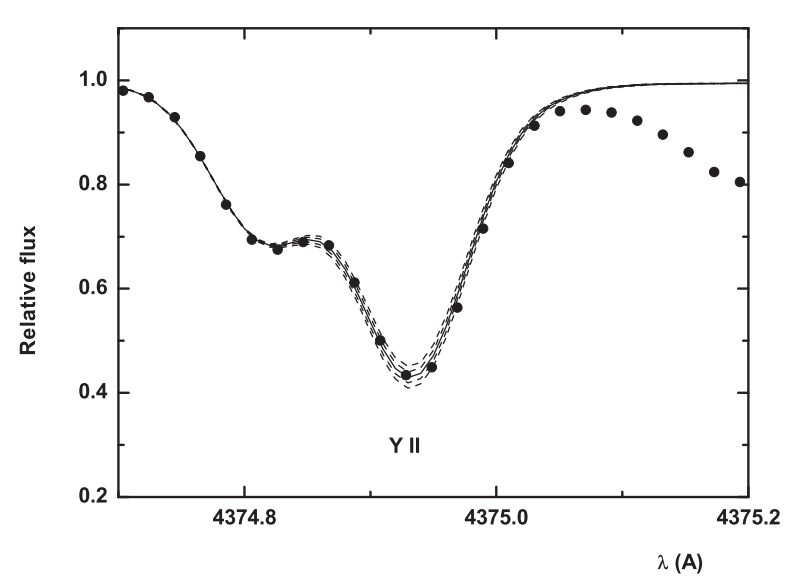

Figure 3. Observed (dots) and calculated (solid and dashed lines) spectra in the region of Y II line for HD22879, the change in the $\mathrm{Y}$ abundance is 0.05 dex.

Table 8. Used solar abundance derived by us and other authors and compared with phosphoric abundance by Asplund et al. (2009).

\begin{tabular}{|c|c|c|}
\hline Species & $\log \mathrm{A}$ (our definitions + ) & Asplund et al. (2009) \\
\hline $\mathrm{Li} \mathrm{I}$ & $1.10(1)$ & $1.05 \pm 0.10$ \\
\hline $\mathrm{O}_{\mathrm{I}}$ & $8.71 \pm 0.05(7)$ & $8.69 \pm 0.05$ \\
\hline $\mathrm{Na} \mathrm{I}$ & $6.25 \pm 0.04(8)$ & $6.24 \pm 0.04$ \\
\hline $\mathrm{Mg}_{\mathrm{I}}$ & $7.54 \pm 0.03(18)$ & $7.60 \pm 0.04$ \\
\hline $\mathrm{Al} \mathrm{I}$ & $6.43 \pm 0.04(9)$ & $6.45 \pm 0.03$ \\
\hline Si I & $7.52 \pm 0.08$ & $7.51 \pm 0.03$ \\
\hline $\mathrm{Ca} \mathrm{I}$ & $6.31 \pm 0.04(45)$ & $6.34 \pm 0.04$ \\
\hline Sc II & $3.06 \pm 0.03(8)$ & $3.15 \pm 0.04$ \\
\hline Ti I & $4.92 \pm 0.05(23)$ & $4.95 \pm 0.05$ \\
\hline Ti II & $5.01 \pm 0.01$ & - \\
\hline$<\mathrm{Ti}>$ & 4.97 & $4.95 \pm 0.05$ \\
\hline $\mathrm{V}_{\mathrm{I}}$ & $3.96 \pm 0.06(25)$ & $3.93 \pm 0.08$ \\
\hline $\mathrm{Cr}$ I & $5.56 \pm 0.08(20)$ & $5.64 \pm 0.04$ \\
\hline Cr II & $5.74 \pm 0.10(7)$ & $5.64 \pm 0.04$ \\
\hline$<\mathrm{Cr}>$ & 5.65 & $5.64 \pm 0.04$ \\
\hline Mn I & $5.22 \pm 0.08(11)$ & $5.43 \pm 0.05$ \\
\hline $\mathrm{Fe} \mathrm{I}$ & $7.50 \pm 0.10(140)$ & $7.50 \pm 0.04$ \\
\hline Fe II & $7.50 \pm 0.11(13)$ & - \\
\hline Co I & $4.96 \pm 0.10(15)$ & $4.99 \pm 0.07$ \\
\hline $\mathrm{Ni} I$ & $6.20 \pm 0.07(32)$ & $6.22 \pm 0.04$ \\
\hline $\mathrm{Cu} \mathrm{I}$ & $4.06 \pm 0.04$ & $4.19 \pm 0.04$ \\
\hline Zn I & $4.54 \pm 0.05$ & $4.56 \pm 0.05$ \\
\hline $\mathrm{Sr}$ II & $2.92 \pm 0.07(5)$ & $2.87 \pm 0.07$ \\
\hline $\mathrm{Y}_{\mathrm{II}}$ & $2.07 \pm 0.05(5)$ & $2.21 \pm 0.05$ \\
\hline $\mathrm{Zr}$ II & $2.60 \pm 0.03$ & $2.58 \pm 0.04$ \\
\hline $\mathrm{Ba} \mathrm{II}$ & $2.17 \pm 0.05(4)$ & $2.18 \pm 0.09$ \\
\hline La II & $1.10 \pm 0.06(5)$ & $1.10 \pm 0.04$ \\
\hline Pr II & $0.81 \pm 0.00$ & $0.72 \pm 0.04$ \\
\hline $\mathrm{Nd}$ II & $1.46 \pm 0.05(4)$ & $1.42 \pm 0.04$ \\
\hline Sm II & 1.00 (Lawler et al. 2006) & $0.96 \pm 0.04$ \\
\hline Eu II & $0.42(4129)$ & $0.52 \pm 0.04$ \\
\hline Eu I & $0.51(6645)$ & $0.52 \pm 0.04$ \\
\hline Gd I & 1.11 (Den Hartog et al. 2006) & $1.07 \pm 0.04$ \\
\hline
\end{tabular}

The abundances of the investigated elements were determined by differential analysis relative to the solar abundances. Solar abundances were calculated using the solar EWs, measured in the spectra of the Moon and asteroids; they were also estimated using the SOPHIE spectrograph and the oscillator strengths log $g f$ adopted from the VALD data base (Kupka et al. 1999). The adopted solar abundance is presented in Table 8 . 
Table 9. Lithium abundance.

\begin{tabular}{lcccccc}
\hline HD & $\begin{array}{c}\log \mathrm{A}(\mathrm{Li}) \\
\text { Our }\end{array}$ & $\begin{array}{c}\text { up(our) } \\
\end{array}$ & $\begin{array}{c}- \\
\text { ( }\end{array}$ & - up $_{1}$ & 2 & 3 \\
\hline 6582 & - & 0.20 & - & 0.4 & - & - \\
6833 & - & -0.20 & - & - & - & - \\
19445 & 2.10 & - & 2.26 & - & 2.10 & 2.25 \\
22879 & 1.50 & - & 1.59 & - & - & 1.44 \\
84937 & 2.31 & - & 2.31 & - & - & 2.4 \\
103095 & 0.51 & - & - & - & - & 0.42 \\
170153 & 2.37 & - & 2.41 & - & - & - \\
216143 & - & -0.50 & - & - & - & - \\
221170 & - & -0.40 & - & - & - & - \\
224930 & - & 0.30 & - & -0.51 & - & - \\
\hline
\end{tabular}

Notes: up(our) - the upper limit of $\mathrm{Li}$ abundance, 1 - Li abundance and up 1 - the upper limit of Li abundance (Ramírez et al. 2012); 2 - Roederer et al. (2014a); 3 - Fulbright (2000).
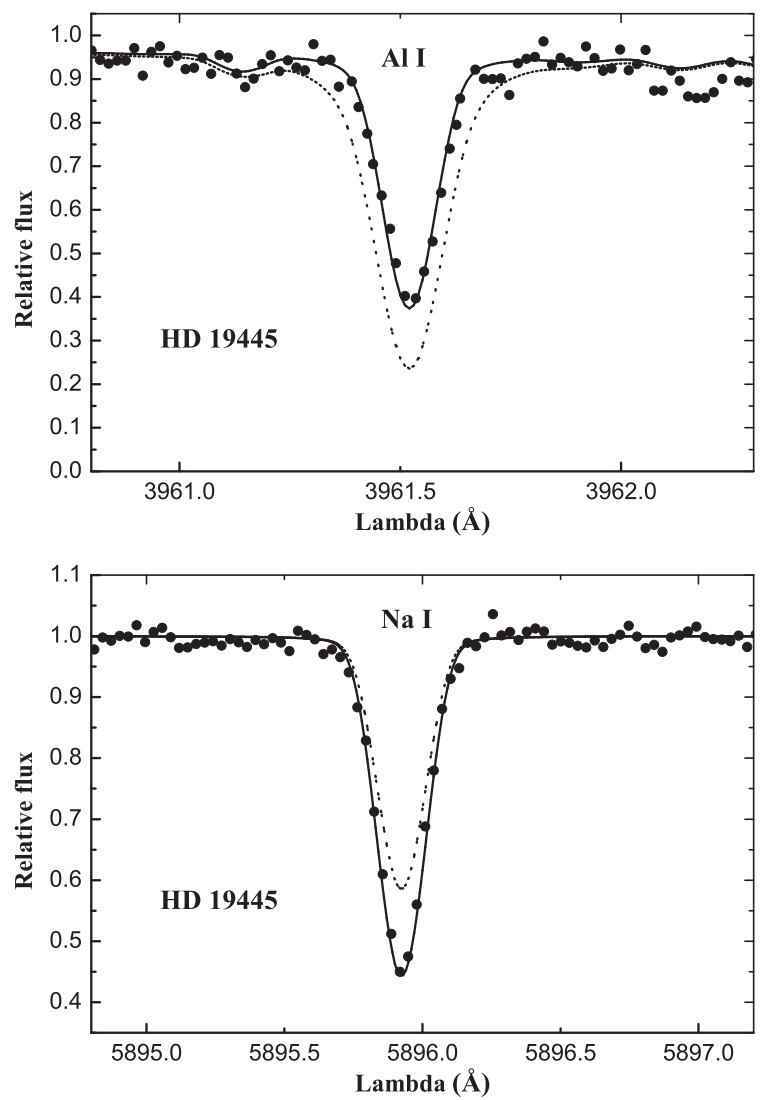

Figure 4. Observed (dots) and calculated (solid - NLTE and dashed - LTE lines) spectra in the region of $\mathrm{Al} \mathrm{I}$ and $\mathrm{Na}$ I lines for HD19445.

\subsection{Lithium abundance}

The Li abundances in the investigated stars were obtained by fitting the observational profiles to the synthetic spectra that were computed by the STARSP LTE spectral synthesis code, developed by Tsymbal (1996). Considering the wide temperature and metallicity ranges of the target stars, we used the detailed list of the atomic and molecular lines in the region of the ${ }^{7} \mathrm{Li} 6707 \AA$ line (Mishenina \& Tsymbal 1997). The comparison of the $\mathrm{Li}$ abundance determinations with the results obtained by other authors is given in Table 9. We can see from this table that our Li determinations are in good agreement with ones of the others authors.

\subsection{NLTE abundance determinations}

The $\mathrm{Na}, \mathrm{Mg}, \mathrm{Al}, \mathrm{Ca}, \mathrm{Sr}$, and $\mathrm{Ba}$ abundances were computed under NLTE approximation with a version of MULTI (Carlsson 1986), modified by S. Korotin (Korotin, Andrievsky \& Luck 1999). The model of $\mathrm{Na}$ atom consists of 27 levels of $\mathrm{Na}$ I and the ground level of $\mathrm{Na}$ I. We considered the radiative transitions between the first 20 levels of $\mathrm{Na}$ I and the ground level of Na II. Transitions between the remaining levels were used only in the equations of particle number conservation. In the linearization procedure, $46 \mathrm{~b}-\mathrm{b}$ and $20 \mathrm{~b}-\mathrm{f}$ transitions were included. We employed the model of $\mathrm{Mg}$ atom consisting of 97 levels: 84 levels of $\mathrm{Mg}$ I, 12 levels of $\mathrm{Mg}$ II, and a ground state of $\mathrm{Mg}$ III. Within the described system of the Mg atom levels, we considered the radiative transitions between the first 59 levels of $\mathrm{Mg}$ I and ground level of $\mathrm{Mg}$ II. Transitions between the rest levels were not taken into account and were used only in the equations of particle number conservation. A more detailed description of these computations can be found in Mishenina et al. (2004). To derive the NLTE Al abundances, we used the $\mathrm{Al}$ I lines at 3944, 3961, 5557.06, 6696.02, and 6698.67 $\AA \AA$. Our Al atomic model is described in detail in Andrievsky et al. (2008). This model atom consists of 76 levels of $\mathrm{Al}$ I and 13 levels of $\mathrm{Al}$ II. Our model of $\mathrm{Ca}$ atom consists of 70 levels of $\mathrm{Ca}$ I, 38 levels of $\mathrm{Ca}$ II, and the ground state of $\mathrm{Ca}$ III were taken into account; in addition, more than 300 levels of $\mathrm{Ca}$ I and $\mathrm{Ca}$ II were included to keep the condition of the particle number conservation in LTE. The information about the adopted oscillator strengths, photoionization cross-sections, collisional rates, and broadening parameters can be found in Spite et al. (2012). For the analysis, we use $45 \mathrm{Ca}$ lines in the visible spectrum. In our analysis, an Sr ion model includes 44 low levels of Sr II with $n \leqq 12$ and $1 \leqq 4$ and the ground level of $\mathrm{Sr}$ III. It also accounts for the fine splitting under the terms $4 \mathrm{~d} 2 \mathrm{D}$ and $5 \mathrm{p} 2 \mathrm{P}^{0}$. That is why we included $24 \mathrm{Sr}$ I levels only into the equation of particle number conservation. A more detailed description of the model atom can be found in Andrievsky et al. (2011). The lines from the blue section of the spectrum, such as resonance lines 4077 and $4215 \AA$, as well as subordinate line $4161 \AA$, were used in this study. Our Ba model contains 31 levels of Ba I, 101 levels of Ba II with $n<50$, and the ground level of Ba III ion. 91 bound-bound transitions between the first 28 levels of Ba II ( $n<12$ and $l<5$ ) were computed in detail. The odd Ba isotopes have HFS of their levels and, thus, several HFS components for each line (Rutten 1978). The information about the adopted oscillator strengths, photoionization cross-sections, collisional rates, and broadening parameters can be found in Andrievsky et al. (2009). The spectrum synthesis fitting of the $\mathrm{Al}$ and $\mathrm{Na}$ lines to the observed profiles is presented in Fig. 4. The abundance determinations for the studied elements are given in Table 10 and are presented in Fig. 5.

For comparison, stellar data observed in the Galaxy are shown from the following references: Reddy et al. (2006), Aoki \& Honda (2008), Roederer et al. (2009, 2014b), Hansen et al. (2012), Ishigaki, Chiba \& Aoki (2012), Ishigaki, Aoki \& Chiba (2013), Cohen et al. (2013), Bensby et al. (2014), Hinkel et al. (2014), and Battistini \& Bensby $(2015,2016)$. The astrophysical implications and discussion based on these observations are given in Section 6.

\subsection{Errors in abundance determinations}

As can be seen from Table 10, the $\mathrm{O}$ abundance has the largest error, ranging between 0.10 and 0.2 dex, this being due to the $\mathrm{O}$ weak lines that we used. The best measured abundances of $\mathrm{Cr}, \mathrm{Fe}$, $\mathrm{Mn}$, the errors are between 0.03 and 0.11 dex. To determine the 
Table 10. Elemental abundance of our target stars.

\begin{tabular}{|c|c|c|c|c|c|c|c|c|c|c|}
\hline HD & 6582 & 6833 & 19445 & 22879 & 84937 & 103095 & 170153 & 216143 & 221170 & 224930 \\
\hline$[\mathrm{Fe} / \mathrm{H}]$ & -0.83 & -0.77 & -2.16 & -0.91 & -2.24 & -1.35 & -0.61 & -2.26 & -2.26 & -0.79 \\
\hline$\sigma, \pm$ & 0.08 & 0.07 & 0.10 & 0.07 & 0.06 & 0.07 & 0.08 & 0.07 & 0.06 & 0.06 \\
\hline$[\mathrm{O} / \mathrm{Fe}]$ & 0.74 & 0.08 & & 0.71 & & 0.73 & 0.05 & 0.46 & 0.46 & \\
\hline$\sigma, \pm$ & 0.20 & 0.10 & & 0.20 & & 0.20 & 0.20 & 0.18 & 0.18 & \\
\hline$[\mathrm{Na} / \mathrm{Fe}]$ & -0.02 & -0.37 & -0.23 & -0.02 & -0.23 & -0.30 & -0.04 & -0.27 & -0.27 & 0.06 \\
\hline$\sigma, \pm$ & 0.12 & 0.14 & 0.15 & 0.12 & 0.14 & 0.12 & 0.12 & 0.18 & 0.18 & 0.10 \\
\hline$[\mathrm{Mg} / \mathrm{Fe}]$ & 0.41 & 0.04 & 0.47 & 0.32 & 0.42 & 0.29 & 0.21 & 0.33 & 0.33 & 0.49 \\
\hline$\sigma, \pm$ & 0.10 & 0.10 & 0.10 & 0.10 & 0.10 & 0.10 & 0.10 & 0.10 & 0.15 & 0.10 \\
\hline$[\mathrm{Al} / \mathrm{Fe}]$ & 0.32 & -0.30 & 0.19 & 0.29 & -0.19 & 0.20 & 0.22 & -0.12 & -0.22 & 0.31 \\
\hline$\sigma, \pm$ & 0.15 & 0.15 & 0.15 & 0.15 & 0.15 & 0.22 & 0.22 & 0.18 & 0.18 & 0.22 \\
\hline$[\mathrm{Si} / \mathrm{Fe}]$ & 0.31 & 0.16 & 0.66 & 0.30 & 0.64 & 0.20 & 0.14 & 0.44 & 0.56 & 0.26 \\
\hline$\sigma, \pm$ & 0.08 & 0.10 & 0.16 & 0.11 & 0.20 & 0.12 & 0.09 & 0.14 & 0.11 & 0.09 \\
\hline$[\mathrm{Ca} / \mathrm{Fe}]$ & 0.24 & 0.32 & 0.36 & 0.26 & 0.46 & 0.28 & 0.14 & 0.30 & 0.46 & 0.37 \\
\hline$\sigma, \pm$ & 0.10 & 0.12 & 0.10 & 0.10 & 0.13 & 0.10 & 0.10 & 0.10 & 0.10 & 0.10 \\
\hline$[\mathrm{Sc} / \mathrm{Fe}]$ & 0.29 & -0.07 & & 0.27 & 0.05 & 0.19 & 0.09 & -0.04 & 0.10 & 0.25 \\
\hline$\sigma, \pm$ & 0.03 & 0.04 & & 0.02 & 0.03 & 0.04 & 0.03 & 0.04 & 0.05 & 0.04 \\
\hline$[\mathrm{Ti} / \mathrm{Fe}]$ & 0.35 & 0.09 & 0.30 & 0.30 & 0.47 & 0.38 & 0.07 & 0.04 & 0.14 & 0.32 \\
\hline$\sigma, \pm$ & 0.03 & 0.06 & 0.05 & 0.06 & 0.03 & 0.07 & 0.04 & 0.05 & 0.05 & 0.04 \\
\hline [V/Fe] & 0.16 & 0.01 & 0.06 & 0.14 & & 0.22 & 0.15 & -0.06 & 0.11 & 0.22 \\
\hline$\sigma, \pm$ & 0.09 & 0.12 & 0.12 & 0.14 & & 0.11 & 0.15 & 0.15 & 0.15 & 0.13 \\
\hline$[\mathrm{Cr} / \mathrm{Fe}]$ & -0.04 & -0.04 & -0.15 & -0.06 & -0.01 & -0.01 & -0.07 & -0.26 & -0.30 & 0.07 \\
\hline$\sigma, \pm$ & 0.05 & 0.07 & 0.11 & 0.08 & 0.08 & 0.08 & 0.06 & 0.04 & 0.03 & 0.06 \\
\hline$[\mathrm{Mn} / \mathrm{Fe}]$ & -0.10 & -0.38 & -0.29 & -0.25 & & -0.18 & -0.08 & -0.41 & -0.35 & -0.10 \\
\hline$\sigma, \pm$ & 0.07 & 0.03 & 0.08 & 0.03 & & 0.02 & 0.04 & 0.03 & 0.01 & 0.09 \\
\hline$[\mathrm{Co} / \mathrm{Fe}]$ & 0.18 & -0.15 & 0.13 & 0.22 & 0.16 & 0.08 & 0.09 & 0.12 & 0.00 & 0.15 \\
\hline$\sigma, \pm$ & 0.08 & 0.11 & 0.07 & 0.11 & 0.03 & 0.12 & 0.15 & 0.13 & 0.08 & 0.11 \\
\hline$[\mathrm{Ni} / \mathrm{Fe}]$ & 0.00 & -0.12 & 0.14 & 0.07 & 0.09 & -0.01 & 0.04 & -0.02 & 0.01 & 0.10 \\
\hline$\sigma, \pm$ & 0.07 & 0.07 & 0.14 & 0.10 & 0.05 & 0.09 & 0.10 & 0.08 & 0.08 & 0.08 \\
\hline$[\mathrm{Cu} / \mathrm{Fe}]$ & 0.08 & -0.54 & & -0.04 & & -0.28 & -0.13 & -0.60 & -0.63 & 0.06 \\
\hline$\sigma, \pm$ & 0.05 & 0.04 & & 0.04 & & 0.11 & 0.06 & & & \\
\hline$[\mathrm{Zn} / \mathrm{Fe}]$ & 0.25 & -0.01 & 0.20 & 0.16 & 0.10 & 0.10 & 0.06 & 0.19 & 0.22 & 0.27 \\
\hline$\sigma, \pm$ & 0.09 & 0.06 & 0.13 & 0.04 & 0.00 & 0.06 & 0.07 & 0.05 & 0.03 & 0.03 \\
\hline$[\mathrm{Sr} / \mathrm{Fe}]$ & -0.01 & -0.14 & 0.02 & 0.03 & 0.07 & -0.08 & 0.06 & -0.04 & -0.08 & 0.05 \\
\hline$\sigma, \pm$ & 0.12 & 0.15 & 0.15 & 0.18 & 0.15 & 0.12 & 0.12 & 0.18 & 0.18 & 0.15 \\
\hline$[\mathrm{Y} / \mathrm{Fe}]$ & 0.07 & -0.21 & -0.14 & 0.12 & & 0.02 & -0.06 & -0.01 & -0.17 & 0.05 \\
\hline$\sigma, \pm$ & 0.05 & 0.09 & 0.06 & 0.05 & & 0.05 & 0.09 & 0.09 & 0.09 & 0.04 \\
\hline$[\mathrm{Zr} / \mathrm{Fe}]$ & 0.33 & 0.07 & & 0.35 & & 0.35 & 0.1 & 0.12 & 0.32 & 0.25 \\
\hline$\sigma, \pm$ & 0.10 & 0.07 & & 0.02 & & 0.04 & 0.12 & 0.10 & 0.06 & 0.07 \\
\hline$[\mathrm{Ba} / \mathrm{Fe}]$ & 0.05 & 0.05 & -0.12 & 0.09 & 0.13 & 0.05 & 0.23 & -0.28 & -0.12 & -0.01 \\
\hline$\sigma, \pm$ & 0.07 & 0.10 & 0.15 & 0.10 & 0.10 & 0.07 & 0.07 & 0.12 & 0.10 & 0.12 \\
\hline$[\mathrm{La} / \mathrm{Fe}]$ & 0.23 & 0.10 & & 0.3 & & 0.28 & 0.21 & 0.07 & 0.31 & 0.07 \\
\hline$\sigma, \pm$ & 0.05 & 0.06 & & 0.07 & & 0.04 & 0.10 & 0.12 & 0.08 & 0.10 \\
\hline$[\mathrm{Pr} / \mathrm{Fe}]$ & & 0.02 & & 0.3 & & & & 0.15 & 0.39 & 0.22 \\
\hline$\sigma, \pm$ & & 0.07 & & & & & & 0.04 & 0.10 & 0.03 \\
\hline$[\mathrm{Nd} / \mathrm{Fe}]$ & 0.11 & 0.11 & & 0.12 & & 0.23 & 0.1 & 0.03 & 0.28 & 0.06 \\
\hline$\sigma, \pm$ & 0.06 & 0.06 & & 0.05 & & 0.07 & 0.05 & 0.08 & 0.05 & 0.09 \\
\hline$[\mathrm{Sm} / \mathrm{Fe}]$ & & & & & & & & 0.18 & 0.45 & \\
\hline$\sigma, \pm$ & & & & & & & & 0.03 & 0.03 & \\
\hline$[\mathrm{Eu} / \mathrm{Fe}]_{4129}$ & 0.35 & 0.31 & & 0.43 & & 0.51 & 0.21 & 0.36 & 0.59 & 0.29 \\
\hline$[\mathrm{Eu} / \mathrm{Fe}]_{6645}$ & 0.34 & 0.44 & & & & & & 0.48 & 0.71 & \\
\hline$[\mathrm{Gd} / \mathrm{Fe}]$ & & & & & & & & 0.18 & 0.47 & \\
\hline$\sigma, \pm$ & & & & & & & & 0.05 & 0.07 & \\
\hline
\end{tabular}

systematic errors in the elemental abundance resulting from uncertainties in the atmospheric parameter determinations, we derived the elemental abundance of two stars with different stellar parameters, HD170153 $\left(T_{\text {eff }}=6170 \mathrm{~K}, \log g=4.25, V_{\mathrm{t}}=0.7\right.$, and $[\mathrm{Fe} / \mathrm{H}]$ $=-0.61)$ and $\operatorname{HD} 221170\left(T_{\mathrm{eff}}=4415 \mathrm{~K}, \log g=1.05, V_{\mathrm{t}}=1.9\right.$, and $[\mathrm{Fe} / \mathrm{H}]=-2.26$ ) for several models with modified parameters $\left(\Delta T_{\text {eff }}= \pm 100 \mathrm{~K}, \Delta \log g= \pm 0.2\right.$, and $\left.\Delta V_{\mathrm{t}}= \pm 0.1\right)$. The abundance variations with the modified parameters, the fitting errors for the computed and observed spectral line profiles $(0.03 \mathrm{dex})$ and total error (tot+), are given in Table 11. The maximum contribution to the error is introduced by $T_{\text {eff }}$ when neutral atom lines are used for the abundance determination and by $\log g$, if the lines of ionized atoms are used. Total error due to parameter uncertainties and the measured of the spectra varies from $0.05-0.11$ dex for the hot and more metallicity stars and to $0.06-0.18$ dex for the cold metal-poor stars, for $[\mathrm{Fe} / \mathrm{H}]$, it is from 0.08 to 0.12 dex, respectively. 


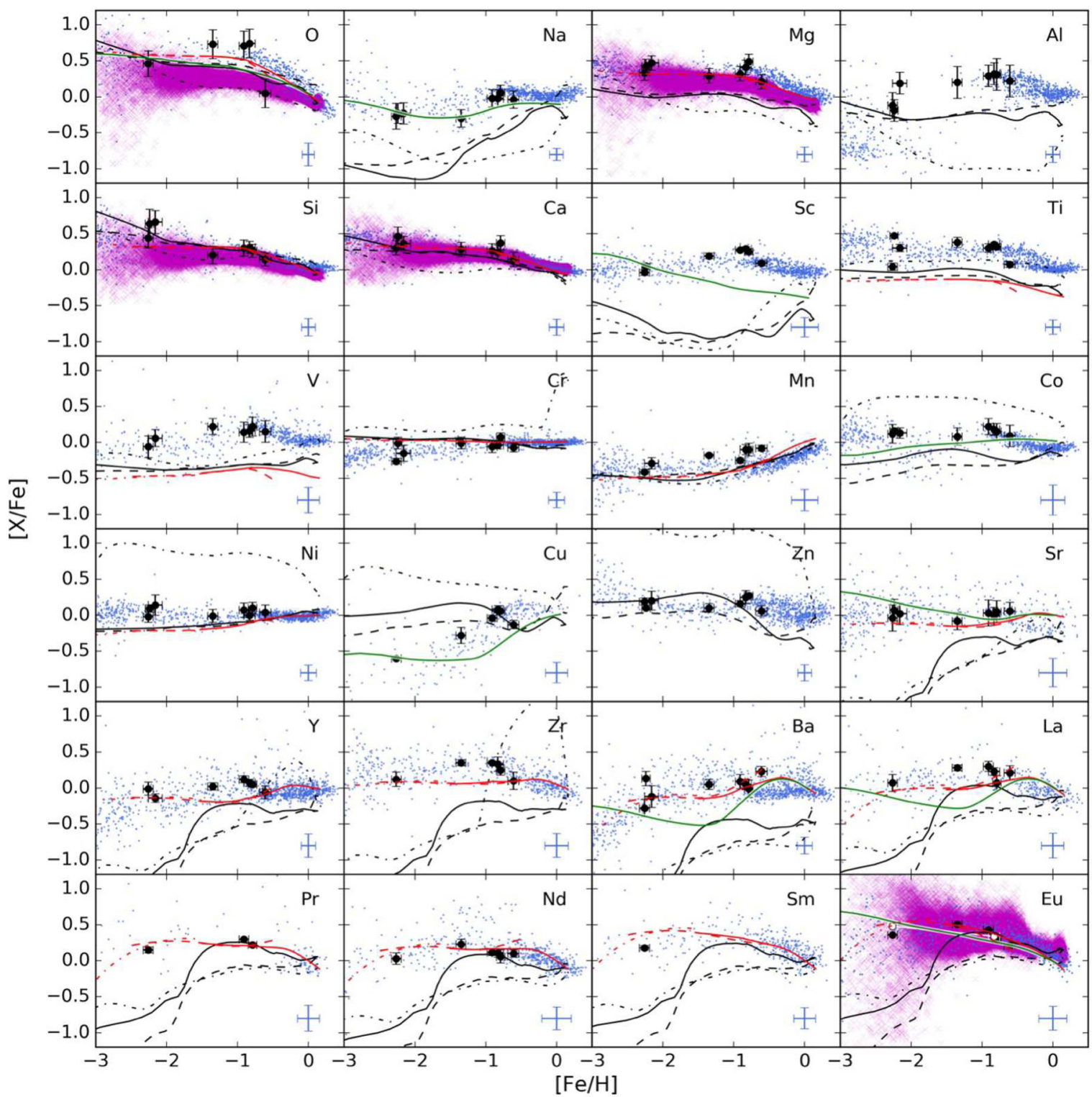

Figure 5. The elemental abundances $[\mathrm{X} / \mathrm{Fe}]$ with respect to $[\mathrm{Fe} / \mathrm{H}]$ is shown for our target stars, in comparison with a large sample of stellar observations in the galaxy, and with predictions from different GCE codes. Our elemental abundances were presented as points with error bars corresponding to the results in Table 10 (note, not all stars have a determined abundance of all investigated elements). The observation data from the literature are marked as blue dots (Aoki \& Honda 2008; Roederer et al. 2009, 2014b; Hansen et al. 2012; Ishigaki et al. 2012, 2013; Cohen et al. 2013; Bensby et al. 2014; Battistini \& Bensby 2015, 2016). Additional data have been used for $\mathrm{Cu}$ (Reddy et al. 2006) and Pr (Hinkel et al. 2014). Black lines have been produced by omEGA, a one-zone model (solid and dashed lines correspond to the massive star yields of West \& Heger (private communication) using the Ertl et al. (2016) and the no-cutoff prescriptions for the stellar remnant masses, respectively. The black dotted lines represent NUGRID Set 1 extension massive star yields. The GCE model predictions by Bisterzo et al. (2014) are shown with red lines (solid line - thin disc, dashed line - thick disc, and dashed-dotted line - halo). The green solid line corresponds to the model predictions associated with the solar neighbourhood chemical evolution model described by Hughes et al. (2008), realized with the GETOOL software package. Results from the ICE code (Wehmeyer, Pignatari \& Thielemann 2015) are shown with magenta crosses.

The comparison of our abundance determinations (1) with those from Jofré et al. (2015), (2) are presented in Table 12 for the stars using as Gaia benchmark.

The mean values of $\langle\Delta[\mathrm{El} / \mathrm{Fe}]>$ are equal to 0.04 \pm 0.05 (HD6582), $\quad-0.05 \quad \pm 0.05$ (HD22879), $\quad-0.09 \quad \pm 0.12$ (HD84937), and $0.14 \pm 0.13$ (HD103095). The greatest shift and spread are due to the difference in temperature obtained in these two studies.

The mean differences of abundance values (for this paper and the work of other authors) and rms deviations $<\Delta[\mathrm{El} / \mathrm{Fe}]>$ are in Table 13 .
As can be seen from Table 13, there is good agreement between our results and those of other authors. The largest discrepancies correspond to differences between our stellar parameters with respect to those from the studies, by Fulbright (2000) for HD6833 and Roederer et al. (2014a) for HD19445. It should be noted that other stars' characterized values from Fulbright (2000) corroborate with those in this study within the stated error definitions. The star HD6833 is a star with $\mathrm{CN}$-weak molecular lines which has no scaled solar chemical composition, but is characterized by the $\mathrm{Na}$ and $\mathrm{Al}$ deficit relative to the $\mathrm{Mg}$ and $\mathrm{O}$ abundances. If we compare the abundances of these elements obtained in this study and 
Table 11. Abundance errors due to atmospheric parameter uncertainties as examples of stars with different values of stellar parameters: HD170153 $\left(T_{\text {eff }}=6170 \mathrm{~K}, \log g=4.25, V_{\mathrm{t}}=0.7 \mathrm{~km} \mathrm{~s}^{-1}\right.$, and $\left.[\mathrm{Fe} / \mathrm{H}]=-0.61\right)$ and $\mathrm{HD} 221170\left(T_{\mathrm{eff}}=4415 \mathrm{~K}, \log g=1.05, V_{\mathrm{t}}=1.9 \mathrm{~km} \mathrm{~s}{ }^{-1}\right.$, and $\left.[\mathrm{Fe} / \mathrm{H}]=-2.26\right)$.

\begin{tabular}{|c|c|c|c|c|c|c|c|c|c|c|}
\hline \multirow[b]{2}{*}{ AN } & \multirow[b]{2}{*}{ El } & \multicolumn{2}{|l|}{ HD170153 } & \multirow[b]{2}{*}{$\Delta V_{\mathrm{t}}+$} & \multirow[b]{2}{*}{ tot+ } & \multirow{2}{*}{1} & \multicolumn{2}{|l|}{ HD221170 } & \multirow[b]{2}{*}{$\Delta V_{\mathrm{t}}+$} & \multirow[b]{2}{*}{ tot +} \\
\hline & & $\Delta T_{\text {eff }}+$ & $\Delta \log g+$ & & & & $\Delta T_{\mathrm{eff}}+$ & $\Delta \log g+$ & & \\
\hline 11 & $\mathrm{Na} I$ & 0.04 & -0.02 & -0.01 & 0.05 & | & 0.08 & -0.02 & -0.03 & 0.09 \\
\hline 12 & $\mathrm{Mg}_{\mathrm{I}}$ & 0.04 & -0.03 & -0.01 & 0.05 & i & 0.07 & -0.03 & -0.05 & 0.09 \\
\hline 13 & $\mathrm{Al}$ I & 0.04 & 0.05 & 0.00 & 0.06 & i & 0.07 & -0.02 & -0.02 & 0.08 \\
\hline 14 & $\mathrm{Si}$ I & 0.05 & 0.04 & -0.01 & 0.06 & i & 0.04 & 0.00 & -0.04 & 0.06 \\
\hline 20 & $\mathrm{Ca} \mathrm{I}$ & 0.07 & -0.04 & -0.03 & 0.09 & i & 0.11 & -0.04 & -0.07 & 0.14 \\
\hline 23.01 & Sc II & 0.03 & 0.08 & -0.01 & 0.09 & i & 0.02 & 0.07 & -0.01 & 0.07 \\
\hline 22 & Ti I & 0.08 & -0.01 & -0.02 & 0.08 & | & 0.18 & -0.02 & -0.01 & 0.18 \\
\hline 22.01 & Ti II & 0.03 & 0.07 & -0.03 & 0.08 & i & 0.02 & 0.06 & -0.01 & 0.06 \\
\hline 23 & $\mathrm{~V}_{\mathrm{I}}$ & 0.09 & 0.00 & -0.02 & 0.09 & 1 & 0.18 & -0.03 & -0.01 & 0.18 \\
\hline 24 & $\mathrm{Cr}_{\mathrm{I}}$ & 0.09 & -0.02 & -0.04 & 0.10 & i & 0.17 & -0.03 & -0.04 & 0.18 \\
\hline 24.01 & $\mathrm{Cr}_{\text {II }}$ & 0.00 & 0.07 & -0.03 & 0.08 & i & -0.03 & 0.07 & -0.02 & 0.08 \\
\hline 25 & Mn I & 0.06 & 0.00 & -0.01 & 0.06 & 1 & 0.12 & -0.02 & 0.00 & 0.12 \\
\hline 26 & $\mathrm{Fe} I$ & 0.07 & -0.02 & -0.03 & 0.08 & | & 0.12 & -0.02 & -0.02 & 0.12 \\
\hline 26.01 & $\mathrm{Fe}$ II & 0.00 & 0.06 & -0.04 & 0.07 & I & -0.03 & 0.07 & -0.02 & 0.08 \\
\hline 27 & Co I & 0.09 & -0.03 & -0.05 & 0.11 & 1 & 0.17 & -0.03 & -0.04 & 0.18 \\
\hline 28 & $\mathrm{Ni}$ I & 0.06 & 0.00 & -0.01 & 0.06 & i & 0.10 & -0.01 & -0.01 & 0.10 \\
\hline 29 & $\mathrm{Cu}$ I & 0.08 & 0.00 & -0.01 & 0.08 & i & 0.14 & -0.02 & -0.01 & 0.14 \\
\hline 30 & $\mathrm{Zn}$ I & 0.05 & 0.02 & -0.04 & 0.07 & 1 & 0.01 & 0.04 & -0.02 & 0.05 \\
\hline 38 & Sr II & 0.05 & 0.02 & -0.02 & 0.06 & 1 & 0.04 & 0.05 & -0.08 & 0.10 \\
\hline 39 & $\mathrm{Y}_{\mathrm{II}}$ & 0.04 & 0.06 & -0.03 & 0.08 & I & 0.03 & 0.07 & -0.01 & 0.08 \\
\hline 40 & $\mathrm{Zr}$ II & 0.04 & -0.02 & 0.08 & 0.09 & i & 0.03 & 0.07 & -0.02 & 0.08 \\
\hline 56 & Ba II & 0.07 & 0.01 & -0.09 & 0.11 & 1 & 0.06 & 0.07 & -0.06 & 0.11 \\
\hline 57 & La II & 0.05 & 0.09 & -0.02 & 0.10 & i & 0.06 & 0.07 & 0.00 & 0.09 \\
\hline 59 & Pr II & 0.04 & 0.07 & 0.00 & 0.08 & i & 0.06 & 0.07 & 0.00 & 0.09 \\
\hline 60 & $\mathrm{Nd}_{\text {II }}$ & 0.04 & 0.07 & 0.00 & 0.08 & I & 0.06 & 0.07 & 0.00 & 0.09 \\
\hline 62 & $\mathrm{Sm}_{\text {II }}$ & 0.04 & 0.07 & 0.00 & 0.08 & I & 0.06 & 0.07 & 0.00 & 0.09 \\
\hline 63 & Eu II & 0.04 & 0.08 & 0.00 & 0.09 & I & 0.07 & 0.08 & 0.04 & 0.11 \\
\hline 64 & Gd II & & & & & 1 & 0.03 & 0.07 & 0.00 & 0.09 \\
\hline
\end{tabular}

Table 12. The comparison of (1) our abundance determinations (2) with those from Jofré et al. (2015), and (3) the difference $\left([\mathrm{El} / \mathrm{H}]_{1}-[\mathrm{El} / \mathrm{H}]_{2}\right)$.

\begin{tabular}{|c|c|c|c|c|c|c|c|c|c|c|c|c|c|c|c|}
\hline \multirow[b]{2}{*}{ El } & \multicolumn{3}{|c|}{ HD 6582} & \multirow{2}{*}{1} & \multicolumn{3}{|c|}{ HD 22879} & | & \multicolumn{3}{|c|}{ HD 84937} & | & \multicolumn{3}{|c|}{ HD 103095} \\
\hline & 1 & 2 & 3 & & 1 & 2 & 3 & I & 1 & 2 & 3 & I & 1 & 2 & 3 \\
\hline$[\mathrm{Fe} / \mathrm{H}]$ & -0.83 & -0.89 & 0.06 & | & -0.91 & -0.85 & -0.06 & I & -2.24 & -2.08 & -0.16 & 1 & -1.35 & -1.34 & -0.01 \\
\hline$[\mathrm{Si} / \mathrm{H}]$ & -0.52 & -0.58 & 0.06 & I & -0.61 & -0.59 & -0.02 & I & -1.60 & -1.73 & 0.13 & I & -1.15 & -1.15 & 0.00 \\
\hline$[\mathrm{Ca} / \mathrm{H}]$ & -0.59 & -0.57 & -0.02 & i & -0.65 & -0.53 & -0.12 & i & -1.78 & -1.67 & -0.12 & i & -1.07 & -1.24 & 0.17 \\
\hline$[\mathrm{Ti} / \mathrm{H}]$ & -0.48 & -0.52 & 0.04 & I & -0.61 & -0.55 & -0.07 & i & -1.77 & -1.66 & -0.11 & i & -0.97 & -1.24 & 0.27 \\
\hline$[\mathrm{Cr} / \mathrm{H}]$ & -0.85 & -0.83 & -0.03 & I & -0.95 & -0.86 & -0.09 & i & -2.23 & -2.23 & -0.00 & i & -1.34 & -1.55 & 0.21 \\
\hline$[\mathrm{Mn} / \mathrm{H}]$ & -0.93 & -1.01 & 0.08 & | & -1.16 & -1.16 & -0.00 & I & - & - & - & | & -1.43 & -1.79 & 0.36 \\
\hline$[\mathrm{Co} / \mathrm{H}]$ & -0.65 & -0.72 & 0.07 & I & -0.69 & -0.74 & 0.05 & I & -2.04 & - & - & I & -1.27 & -1.38 & 0.11 \\
\hline$[\mathrm{Ni} / \mathrm{H}]$ & -0.83 & -0.83 & -0.00 & i & -0.84 & -0.85 & 0.01 & i & -2.15 & -2.06 & -0.09 & i & -1.36 & -1.50 & 0.14 \\
\hline
\end{tabular}

in Fulbright (2000) and Luck (1991) (Table 14), we can see that the ratio of these elements is similar, while the metallicity value is different. The comparison of the EWs of the lines measured by us and by Fulbright (2000), $<$ (EW(fulb) $-\mathrm{EW}($ our $)>=-1.83$ \pm 5.65 , showed a good agreement between the values. The fact that Fulbright (2000) have only used three lines of neutral iron for the HD6833 study, while from 30 to 60 lines were used for other stars in their study, is the most plausible cause for this difference. The shift and scatter of values for HD19445 is due to the difference between the $\mathrm{Al}$ abundance obtained by us $([\mathrm{Al} / \mathrm{Fe}]=0.19)$ and that one by Roederer et al. (2014a) $([\mathrm{Al} / \mathrm{Fe}]=-0.56)$. If the comparison is made without accounting the $\mathrm{Al}$ abundance, we obtained: $<\Delta[\mathrm{El} / \mathrm{Fe}]>=0.08 \pm 0.13$. The difference in the $\mathrm{Al}$ abundance is due to the fact that Roederer et al. (2014a) analysis did not take into account NLTE corrections that at this metallicity is about 0.6 dex for the lines used.

\section{RESULTS AND DISCUSSION}

Our results for different elements are summarized in Fig. 5 and Table 10. Among our target stars, there are two stars with peculiar chemical composition, HD6833, a CN-weak giant and HD221170, a rich $r$-process metal-poor star.

HD6833. For this star, we have a chemical composition that is slightly different from solar scaled: there are under abundances for $\mathrm{Na}, \mathrm{Al}, \mathrm{Mn}$, and $\mathrm{Cu}$; also a small deficit of $\mathrm{Sr}$ and $\mathrm{Y}$; and a significant excess of $\mathrm{Ca}$ and $\mathrm{Eu}$ compared to the Sun. The values of $\mathrm{Ca}, \mathrm{Mn}$, and $\mathrm{Eu}$ correspond to those of these elements at this metallicity, $\mathrm{Na}$ 
Table 13. The comparison of our abundance determinations with those of other authors: $<\Delta[\mathrm{El} / \mathrm{Fe}]>$ is mean differences of abundance values and rms deviations.

\begin{tabular}{lcl}
\hline HD & $<\Delta[\mathrm{El} / \mathrm{Fe}]>$ & \multicolumn{1}{c}{ References } \\
\hline 6582 & $-0.01 \pm 0.10$ & Fulbright (2000) \\
& $0.04 \pm 0.07$ & Gratton et al. (2003) \\
6833 & $-0.15 \pm 0.20$ & Fulbright (2000) \\
19445 & $-0.04 \pm 0.08$ & Fulbright (2000) \\
& $0.12 \pm 0.22$ & Roederer et al. (2014a) \\
& $0.07 \pm 0.17$ & Gratton et al. (2003) \\
22879 & $-0.02 \pm 0.06$ & Fulbright (2000) \\
& $0.07 \pm 0.09$ & Klochkova et al. (2011) \\
& $0.06 \pm 0.12$ & Gratton et al. (2003) \\
84937 & $0.01 \pm 0.10$ & Fulbright (2000) \\
& $0.05 \pm 0.11$ & Gratton et al. (2003) \\
103095 & $0.01 \pm 0.11$ & Fulbright (2000) \\
& $0.09 \pm 0.10$ & Gratton et al. (2003) \\
216143 & $-0.03 \pm 0.16$ & Fulbright (2000) \\
221170 & $0.04 \pm 0.13$ & Fulbright (2000) \\
& $0.00 \pm 0.13$ & Ivans et al. (2006) \\
224930 & $0.00 \pm 0.14$ & Fulbright (2000) \\
& $0.06 \pm 0.09$ & Stonkute et al. (2012) \\
& $0.04 \pm 0.08$ & Gratton et al. (2003) \\
\hline
\end{tabular}

Table 14. For HD6833, the comparison of our $\mathrm{O}, \mathrm{Na}, \mathrm{Mg}$, and $\mathrm{Al}$ abundance determinations with those of other authors: 1 - Fulbright (2000) and 2 - Luck (1991).

\begin{tabular}{lccc}
\hline $\mathrm{El}$ & This work & 1 & 2 \\
\hline$[\mathrm{Fe} / \mathrm{H}]$ & -0.77 & -1.04 & -0.75 \\
{$[\mathrm{O} / \mathrm{Fe}]$} & 0.08 & - & -0.21 \\
{$[\mathrm{Na} / \mathrm{Fe}]$} & -0.37 & -0.06 & -0.43 \\
{$[\mathrm{Mg} / \mathrm{Fe}]$} & 0.04 & 0.45 & 0.15 \\
{$[\mathrm{Al} / \mathrm{Fe}]$} & -0.30 & 0.16 & -0.36 \\
\hline
\end{tabular}

and $\mathrm{Al}$ abundances depart significantly from the general trend. As shown above, the ratio of these element abundances agrees with that obtained by Luck (1991). At the same time, Luck (1991) showed that the $\mathrm{CNO}$ abundance in the $\mathrm{CN}$-weak giants differs only slightly (within the definition error) from that of 'normal' giants, and of giants with G-weak band, and also on standard calculations of stellar evolution. However, they stressed that there is still the problem of a small $\mathrm{C}$ deficit. The distinctive ratio of $\mathrm{O}$ and $\mathrm{Na}, \mathrm{Mg}$ and $\mathrm{Al}$ abundances may serve as a test for theories of stellar evolution (see e.g. Denissenkov \& Weiss 1996; Denissenkov et al. 1998; Prantzos, Charbonnel \& Iliadis 2007; Denissenkov et al. 2015), including the stars with moderate deficit of Fe. Thus, this star with particular enrichment in some elements requires a special study. Therefore, in this study applied to GCE, we exclude this star.

$H D 221170$. It is a well-known halo star with $r$-process enrichment. Also in our study, we took a star HD216143 with the similar parameters as for star HD221170, to compare the chemical composition of these two stars. For HD221170, we have obtained a slight excess of $\mathrm{Eu}$ ( $r$-process element) abundance relative to those for HD216143, and also of other elements formed in neutron capture processes. Overabundance of $r$-process elements in HD221170 is due to anomalous enrichment of pristine material from where the star formed, possibly indicating an incomplete mixing at that time (e.g. Ivans et al. 2006). We have not included HD221170 in our study applied to GCE.

\subsection{Membership of stars to galactic populations}

The necessary and sufficient criterion to classify each star into the thin, thick discs, and halo of the Galaxy does not exist. However, with galactic velocities or orbital elements, metallicity and relative abundance of some chemical elements, it can be attempted to classify each star into its most probable stellar population. For instance, Hawkins et al. (2015) explore the Galactic disc-halo transition region between $-1.20<[\mathrm{Fe} / \mathrm{H}]<-0.55$ and show that may be able to chemically label the Galactic components in a clean and efficient way independent of kinematics using $[\alpha / \mathrm{Fe}],[\mathrm{C}+\mathrm{N} / \mathrm{Fe}],[\mathrm{Al} / \mathrm{Fe}]$, and $[\mathrm{Mg} / \mathrm{Mn}]$. Also, using the total spatial velocity or eccentricity of the orbit, it is possible to distinguish stars from the initial and accreted halo (Venn et al. 2004; Carollo et al. 2010).

Here, we attempt to classify the target stars into the halo and thick disc populations according to their dynamics and abundances. According to their high eccentricity (ecc $>0.8$, see Table 6), HD6833, HD84937, HD103095, and HD216143 are likely halo stars. HD84937 and HD216143 have also $[\mathrm{Fe} / \mathrm{H}]<-2$ which confirms their halo membership. HD6833 has a higher metallicity, $[\mathrm{Fe} / \mathrm{H}]=-0.77$, with a moderate $\alpha$ enhancement, $[\alpha / \mathrm{Fe}]=+0.17$. In this work, the average abundance of $\mathrm{Mg}, \mathrm{Si}$, and $\mathrm{Ca}$ are taken to calculate the $[\alpha / \mathrm{Fe}]$ ratio. The resulting $\alpha$ value is consistent with Nissen \& Schuster (2010), where the 'low- $\alpha$ ' stars are suggested to be accreted from dwarf galaxies. However, HD6833 is a $\mathrm{CN}$-weak star with peculiar chemical composition. According to Hawkins et al. (2015), this is a signature of the accreted halo. HD224930 and HD170153 have more circular orbits confined close to the galactic plane which makes them more likely thick disc stars. HD224930 and HD170153 have V velocities, respectively -76 and $+45 \mathrm{~km} \mathrm{~s}^{-1}$, are not typical of thin disc. In the solar neighbourhood, the thin disc rotates at about $-10 \mathrm{~km} \mathrm{~s}^{-1}$ with respect to the Sun with a typical standard deviation of $\sim 20 \mathrm{~km} \mathrm{~s}^{-1}$ (see for instance Soubiran, Bienaymé \& Siebert 2003). In addition, both stars have $[\mathrm{Fe} / \mathrm{H}]<-0.50$ which is also characteristics of the thick disc. However their membership to the thin disc cannot be totally ruled out. HD224930 has $[\mathrm{Fe} / \mathrm{H}]=-0.79$ and $[\alpha / \mathrm{Fe}]=0.37$ which is also typical of the thick disc. There are four stars (HD6582, HD19445, HD22879, and HD221170) which have orbital parameters compatible either with the halo or the thick disc. HD19445 and HD221170 have a low metallicity, $[\mathrm{Fe} / \mathrm{H}]<-2$, and a high $\alpha$ enhancement $([\alpha / \mathrm{Fe}]>+0.4)$ typical of the halo. HD6582 and HD22879 are intermediate in their kinematical and chemical properties which makes them impossible to classify. The dependence of $[\alpha / \mathrm{Fe}]$ with respect to $[\mathrm{Fe} / \mathrm{H}]$ are shown in Fig. 6. Membership in the galactic populations is given in Table 6.

\subsection{A special element: lithium}

Li is easily destroyed at typical H-burning conditions in stars. On the other hand, it may be produced as a result of extra-mixing processes in AGB stars and red giant branch stars of different initial masses via the Cameron-Fowler transport mechanism (see e.g. Lattanzio \& Forestini 1999; Sackmann \& Boothroyd 1999; Nollett, Busso \& Wasserburg 2003; Denissenkov \& Merryfield 2011; Palmerini et al. 2011). Such non-standard mixing processes are challenging to simulate for baseline onedimensional stellar models, as large differences do exist in theoretical predictions (e.g. Lattanzio et al. 2015). Also because of this high dependence on the local stellar conditions, $\mathrm{Li}$ is a powerful diagnostic for stellar evolution, GCE models and cosmology. Li is made in the big bang (Burbidge et al. 


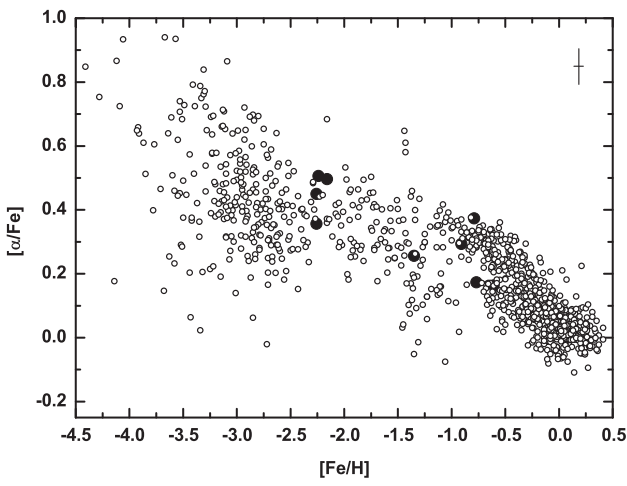

Figure 6. Dependence of $\alpha$ elements abundance on $[\mathrm{Fe} / \mathrm{H}]$. The elements $\mathrm{Mg}, \mathrm{Si}$, and $\mathrm{Ca}$ were taken to calculate the averaged $\alpha$-element abundances. Our data marked as full circles and data of Bensby et al. (2014), Cohen et al. (2013), Ishigaki et al. (2012), and Roederer et al. (2014b) marked as small open circles.

1957). Encouraging results for metal-poor stars which confirm big bang nucleosynthesis simulations were reported in the study by Spite \& Spite (1982). Metal-poor dwarfs studied by Spite \& Spite (1982) showed similar Li abundances with small dispersion. This was referred to as the cosmological Li contribution. A large number of following investigations focused on both the cosmological Li abundance and the dispersion of that value, on both observational and theoretical ground (see Cyburt et al. 2016, and references therein). In particular, a spread of the $\mathrm{Li}$ abundances was later found by Thorburn et al. (1993) and Meléndez et al. (2010). The WMAP mission also confirmed that the cosmological Li abundance differs significantly from observations in metal-poor dwarfs. Among our stars, HD19445 and HD84937 with $[\mathrm{Fe} / \mathrm{H}]<-2.0$ dex have long history of study ${ }^{6} \mathrm{Li} /{ }^{7} \mathrm{Li}$ (e.g. Smith, Lambert \& Nissen 1993; Hobbs \& Thorburn 1994; Cayrel et al. 1999). The isotopic ratio of ${ }^{6} \mathrm{Li} /{ }^{7} \mathrm{Li}$ was proposed as an important indicator of efficiencies of mixing processes in the stellar interior. The Li formation in the solar spots (Livshits 1997) and spallation reactions (Goriely 2008), and in the case of metal-poor stars, also for resolving of cosmological Li problem (e.g. Asplund et al. 2006; Fields 2011). However, recently Lind et al. (2013) found that the observational support for significant ${ }^{6} \mathrm{Li}$ production in the early Universe proposed by Asplund et al. (2006) is substantially weakened by their findings.

In this work, we provide the Li elemental abundance, but we do not provide the ${ }^{6} \mathrm{Li} /{ }^{7} \mathrm{Li}$ ratio. For $\mathrm{Li}$, the isotope shift is small, and in order to confidently determine ${ }^{6} \mathrm{Li} /{ }^{7} \mathrm{Li}$ we would need a spectrum with a resolution of about 100000 and a ratio of $\mathrm{S} / \mathrm{N}$ of 500 . In general, we obtain low Li abundance for stars with $T_{\text {eff }}<5600 \mathrm{~K}$. This supports the hypothesis of the destruction of Li by H-burning depleting the pristine Li concentration (see Table 9 and Fig. 7). From our stellar sample, the star HD 19445 shows an Li abundance $=2.1$ that is 0.08 dex lower than the average $\mathrm{Li}$ abundance. This is the largest variation that we observe, that is within observational errors. For all the other stars, we get variations lower than 0.04 dex.

At present, the GCE of Li in the Galaxy is uncertain, due to the fact that $\mathrm{Li}$ is not only easily destroyed in stellar interiors during the stellar evolution, but can also be produced by stars as mentioned above. To avoid any possible $\mathrm{Li}$ abundance variations caused by stellar evolution, only dwarf stars should be used (with $T_{\text {eff }}>5600 \mathrm{~K}$ and $\log g>3.7$, e.g. Guiglion et al. 2016). We have four stars with such parameters, namely, HD 19445, HD22879, HD84937, and HD170153. Among them there are two stars with $[\mathrm{Fe} / \mathrm{H}]<-1.5$, HD19445 and HD22879, that have the values of $[\mathrm{Fe} / \mathrm{H}]$ and $\log$

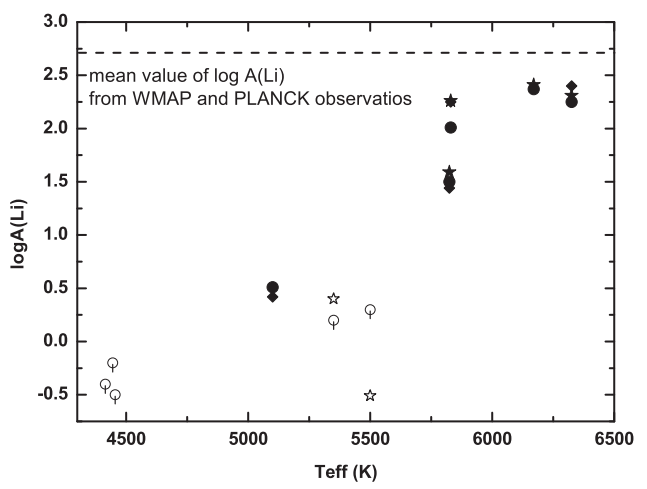

Figure 7. Dependences of $\log \mathrm{A}(\mathrm{Li})$ on $T_{\text {eff }}$. Our Li abundance and the upper limit are marked as full and open circles, respectively. Li abundance and the upper limit are marked as full and open asterisks (Ramírez et al. 2012), and observations by Fulbright (2000) with diamonds. The mean values of $\log \mathrm{A}(\mathrm{Li})$ based on WMAP and Planck observations: 2.65 (Steigman 2007), 2.72 (Cyburt, Fields \& Olive 2008), and 2.66 (Cyburt et al. 2016).

$\mathrm{A}(\mathrm{Li})$ close to these values for the Spite plateau $(\log \mathrm{A}(\mathrm{Li})=2.2)$ found by Spite \& Spite (1982). Taking into account the standard big bang nucleosynthesis model, the primordial $\mathrm{Li}$ abundance is predicted to be $\log \mathrm{A}(\mathrm{Li})=2.6$ (Spergel et al. 2003). Li can be produced in the interstellar medium via spallation by Galactic cosmic rays (GCR) and by different types of stars (see e.g. Guiglion et al. 2016). The chemical evolution of Li in the Milky Way was constructed by Prantzos (2012). According to these results, GCR and primordial nucleosynthesis can produce at most $\sim 30$ per cent of solar $\mathrm{Li}$, but its stellar production is too low to explain the missing Li component.

\subsection{Comparing observations with GCE simulations}

The evolution of the chemical inventory of the galaxy from its early stages (e.g. Sneden, Cowan \& Gallino 2008; Bonifacio et al. 2012; Griffen et al. 2016) until the youngest stellar generations observed in open clusters and associations (e.g. Biazzo et al. 2012; Carraro 2015; Mishenina et al. 2015a) provides an invaluable source of information about the galaxy formation, its evolution and about how real stars work. A consistent interpretation of the evolution of elemental ratios at different metallicities is one of the main task of GCE. GCE models are folding theoretical stellar yields within the fundamental physics equations driving the dynamics of the galaxy. This allows to test theoretical models, its chemical products, and all the different pieces of physics relevant for a given observable. For instance, the observation of $\mathrm{C}$ and $\mathrm{N}$ in old stars ( $\mathrm{C}$ and $\mathrm{N}$ abundances are not provided in this analysis) in the early Galaxy may provide insights about the core-collapse supernova (CCSN) engine, how fast the massive star progenitors were rotating and about ingestion of material between different stellar layers, or about the $\mathrm{C}$ production in AGB stars, that are the most relevant source of $\mathrm{C}$ and $\mathrm{N}$ in the galactic disc and Sun (e.g. Spite et al. 2005; Chiappini et al. 2006; Bonifacio et al. 2015; Pignatari et al. 2015; Frischknecht et al. 2016; Yoon et al. 2016). The lighter $\alpha$-elements $\mathrm{O}$ and $\mathrm{Mg}$ are indicative of the evolution of massive star progenitors (e.g. Thielemann, Nomoto $\&$ Hashimoto 1996), while heavier $\alpha$ elements ( $\mathrm{Si}, \mathrm{Ca}$, and $\mathrm{Ti}$ ) and the Fe group elements are mostly affected by the CCSN explosion at low metallicities (e.g. Woosley \& Weaver 1995; Woosley, Heger \& Weaver 2002; Thielemann et al. 2011b; Nomoto, Kobayashi \& Tominaga 2013) and by SNe Ia once these start to contribute to the galactic chemical inventory (e.g. Hillebrandt et al. 2013, and 
references therein). The chemical evolution of heavy elements allows to constrain theoretical simulations for the $s$-process (e.g. Käppeler et al. 2011), the $r$-process (Thielemann et al. 2011a, and references therein), and for a wide range of less-constrained nucleosynthesis processes, like the $i$-process (e.g. Cowan \& Rose 1977; Herwig et al. 2011; Bertolli et al. 2013; Dardelet et al. 2014; Mishenina et al. 2015a; Jones et al. 2015; Roederer et al. 2016; Hampel et al. 2016) and a zoo of explosive neutrino-wind components from $\mathrm{SNe}$ (e.g. Fröhlich et al. 2006b,a; Qian \& Wasserburg 2008; Farouqi et al. 2009; Roberts, Woosley \& Hoffman 2010; Arcones \& Montes 2011; Hansen \& Primas 2011; Wanajo, Janka \& Kubono 2011; Arcones \& Thielemann 2013; Hansen et al. 2013). However, the results from GCE simulations depend also on the assumptions and simplifications made by the model (e.g. Gibson et al. 2003), and on the theoretical stellar yields adopted.

In Fig. 5, we compared the results obtained from our stellar sample, with observations from other stars in the Milky Way. Within the observational errors, in general our stars show abundance patterns consistent with the average chemical enrichment history of the Milky Way. In the same figure, we also provide a sample of prediction from GCE models, calculated using different codes, assumptions, and stellar yields. Departure of single stars from the average evolutionary trends of elemental ratios may be due to observational errors or peculiar enrichment histories. The stars in our sample follow quite well the average chemical evolution of the Milky Way. In the following part of the section, we therefore compare predictions from different GCE models with the average abundance trends in the galaxy. The conclusions that we will derive also apply to our stellar sample.

Black lines have been produced by OMEGA, a one-zone model that is available online with the NUGRID NUPYCEE chemical evolution package. ${ }^{2}$ This simple code is designed to capture the global trends generated by a set of stellar yields and to provide an easy-to-use platform to test and compare stellar models. It takes into account inflows of primordial gas and galactic outflows driven by star formation (see Côté et al. 2017). The star formation history and the dark matter and gas masses are input parameters in order to mimic the evolution of a specific galaxy, here the Milky Way. OMEGA assumes homogenous mixing but takes into account the delay between star formation and stellar ejecta. Each stellar population formed throughout a simulation, using SYGMA (Stellar Yields for Galactic Modeling Applications, Ritter et al., in preparation), is followed in time by considering their specific age, mass, and metallicity. We refer to Côté et al. (2016b) for more details on the different input parameters for stellar populations and to Côté et al. (2016a) for the numerical setup for the Milky Way.

For OMEGA, we used NUGRID AGB stellar yields for low- and intermediate-mass stars (Pignatari et al. 2016; Ritter et al., in preparation). SNe Ia are included with a delay-time distribution in the form of $t^{-1}$ (Maoz, Mannucci \& Nelemans 2014) and the yields calculated by Thielemann, Nomoto \& Yokoi (1986). The black solid and dashed lines in Fig. 5 have respectively been generated with the massive star yields of West \& Heger (private communication) using the Ertl et al. (2016) and the no-cutoff prescriptions for the stellar remnant masses (see Côté et al. 2016a for more details). The black dotted lines represent NUGRID Set 1 extension massive star yields (Ritter et al., in preparation), using the stellar remnant mass prescription of Fryer et al. (2012), along with the zero-metallicity yields of West \& Heger (private communication). Massive star yields are

${ }^{2}$ https://github.com/NuGrid/NUPYCEE only applied to stars with initial mass between 8 and $30 \mathrm{M}_{\odot}$. We thus assume no ejecta for stars more massive than $30 \mathrm{M}_{\odot}$ (see discussion in Côté et al. 2016b). For the ejection of $r$-process material, we only considered the contribution of neutron star mergers. We used the delay-time distribution of the standard models of Dominik et al. (2012) to distribute the yields as a function of time for each simple stellar population. We assumed that each neutron star merger ejects a total mass of $0.01 \mathrm{M}_{\odot}$ with the $r$-process composition provided by Arnould, Goriely \& Takahashi (2007). Overall, our implementation generates $5.5 \times 10^{-5}$ neutron star merger event per unit of solar mass formed.

The GCE model predictions by Bisterzo et al. (2014) are shown in Fig. 5 with red lines. This code was presented by Travaglio et al. (1999, 2004), and follows the composition of stars, stellar remnants, interstellar matter (atomic and molecular gas), and their mutual interaction, in the three main zones of the Galaxy, halo, thick, and thin discs. The chemical evolution is calculated inside the solar annulus, located $8.5 \mathrm{kpc}$ from the Galactic Centre. The thin disc is divided into independent concentric annuli. The chemical evolution is regulated by the star formation rate (SFR), initial mass function (IMF), and nucleosynthesis yields from different stellar mass ranges and populations. The SFR has been determined self-consistently as the result of aggregation, interacting, and interchanging processes of the interstellar gas, which may occur spontaneously or stimulated by the presence of other stars. The treatment of the elemental matrix and yields have been updated by Bisterzo et al. (2014), as presented here.

Concerning the heavy elements, the $r$-process yields are derived as explained by Travaglio et al. (1999). Because of the large uncertainties affecting the astrophysical site and physical conditions of the $r$-process, the solar $r$-process contribution for elements heavier than $\mathrm{Ba}$ is derived by adopting the $r$-process residuals method (e.g. Arlandini et al. 1999). This method is evaluated by subtracting the $s$-process contributions from the solar abundances $\left(N_{r}=\mathrm{N}_{\odot}-N_{s}\right)$, still providing a good approximation to derive the solar $r$-process abundances from $\mathrm{Ba}$ to $\mathrm{Bi}$. We assumed the $r$-process yields as primary and occurring in CCSN with a limited range of progenitor masses (8-10 $\mathrm{M}_{\odot} \mathrm{SNe}$ ), following the observed decreasing trend of heavy neutron-capture elements in the early Galaxy.

As discussed by Travaglio et al. (2004), we have included an additional primary contribution ([Lighter Element Primary Process (LEPP)] to interpret the trend observed for light neutron-capture elements (as Sr-Y-Zr).

Concerning massive stars, for this work we took the yields from: (1) massive stars from 13 to $30 \mathrm{M}_{\odot}$ from West \& Heger (private communication) with the no-cutoff remnant mass prescription; (2) stars more massive than $30 \mathrm{M}_{\odot}$ and up to $100 \mathrm{M}_{\odot}$ from Limongi \& Chieffi (2012) and Chieffi \& Limongi (2013) (up to Mo); (3) SNe Ia from Travaglio, Hillebrandt \& Reinecke (2005).

The green solid line corresponds to the model predictions associated with the solar neighbourhood chemical evolution model described by Hughes et al. (2008), realized with the GETOOL software package (Fenner \& Gibson 2003). Nucleosynthetic yields were drawn from Woosley \& Weaver (1995), Karakas \& Lattanzio (2007), and Nomoto et al. (1997) for CCSNe, AGB stars, and SNe Ia, respectively. Linear extrapolation of the CCSNe yields from $40 \mathrm{M}_{\odot}$ to $60 \mathrm{M}_{\odot}$ was employed, with a lower mass limit of $0.08 \mathrm{M}_{\odot}$ adopted, with the mass and metallicity interpolation scheme as outlined by Gibson (1997); the distribution of stellar masses employed in the modelling was that described by Kroupa, Tout \& Gilmore (1993). A dual infall framework was used with a rapid initial infall of gas on essentially a free-fell time-scale (50 Myr), referred to 
as the halo phase, followed by a more protracted infall phase on an exponential time-scale of $10.5 \mathrm{Gyr}$ (after a delay of $500 \mathrm{Myr}$ with respect to the halo phase). A conservative star formation law predicated upon the class Schmidt Law was employed with the SFR proportional to the square of the local gas surface density, modulated by an efficiency factor of $0.06 \mathrm{Gyr}^{-1}$. The overall model is constrained to recover a local total mass surface density of $55 \mathrm{M}_{\odot} \mathrm{pc}^{-2}$ in the solar neighbourhood. The $r$-process yields were simply estimated using the residual method, i.e. from the difference between solar and $s$-process predictions (e.g. Arlandini et al. 1999).

Finally, the results for the inhomogeneous chemical evolution model 'ICE' are shown with magenta cross symbols for $\mathrm{O}, \mathrm{Mg}$, $\mathrm{Si}, \mathrm{Ca}$, and $\mathrm{Eu}$. ICE is able to keep track of the intrinsic inhomogeneities in the interstellar medium. In comparison to other GCE models which employ an instantaneous mixing approximation, the inhomogeneities in our model produce a scatter in observed abundances, especially at lower metallicities, before a sufficient number of events cause convergence to average values. The main iteration procedure of one time-step $\left(10^{6} \mathrm{yr}\right)$ can be summarized as follows. Primordial gas is falling into the simulation volume. The SFR is calculated via a Schmidt-Kennicutt law with a power of 1.5. Cells are chosen randomly to trigger star formation, however, higher density cells are favoured. The mass of a newly born star is chosen randomly from a Salpeter IMF (with an integrated slope of -1.35). The newly born stars inherit the abundances of the local interstellar medium. The lifetime of a star is calculated via an age-life expectance relation of the Geneva Stellar Evolution and Nucleosynthesis Group (e.g. Schaller et al. 1992). Dependent on their initial mass, stars which reached the end of their lifetime will either undergo a CCSN event (with stellar yields given by Thielemann, Nomoto \& Hashimoto 1996) or blow off processed matter into the interstellar medium via a planetary nebula. A fraction of $9 \times 10^{-4}$ of all intermediate star binary systems will results in an SNe Ia explosion, with an ejecta composition taken from (Iwamoto et al. 1999, model CDD2 yields). A fraction of $3.8 \times 10^{-4}$ of high-mass star binary systems ends in a neutron star merger event after both stars have independently exploded as CCSNe and an inspiral delay time (or coalescence time) of $10 \mathrm{Myr}$ has passed. The ejecta of these events are taken from Korobkin et al. (2012), following a solar $r$-process distribution. Stars in the surrounding interstellar medium are polluted by the ejecta of the respective nucleosynthesis event.

The main difference to the other models, presented here including $r$-process element yields (e.g. for Eu), is that ICE assumes that in addition a fraction of all CCSNe ( 0.1 per cent) explodes as 'magnetorotationally driven $\mathrm{SNe}$, leading to the formation of magnetars, i.e. neutron stars with magnetic fields beyond $10^{15} \mathrm{G}$ (see Winteler et al. 2012; Nishimura, Takiwaki \& Thielemann 2015, for discussion), and producing $r$-process elements in polar jet ejecta during the explosion. Thus, the main difference is that also an $r$-process source exists related to massive single stars which does not require the delay of binary evolution after individual SN explosions producing $\mathrm{Fe}$ and the merger event producing $r$-process elements.

A detailed description of the chemical evolution model can be found in Wehmeyer et al. (2015). While GETOOL, OMEGA, and threezone models (green, black, and red lines, respectively) represent spatially averaged values of the abundance scatter observed at low metallicities, the approach adopted by ICE model (pink crosses) provides a more realistic view of the local chemical inhomogeneities detected in the interstellar medium at early times. ICE predictions allow to study not only the average trend for a given elemental ratio with metallicity, but also the dispersion at any given time due to local inhomogeneities, before the stellar products are fully mixed.
These inhomogeneities allow to explain the scatter in elemental abundances, especially at low metallicities. With the largest scatter seen in $r$-process elements, the biggest advantage of an inhomogeneous GCE treatment is revealed: when a rare sub-class of $\mathrm{SNe}$ ('magnetorotationally driven $\mathrm{SNe}$ ', with an occurrence rate of probably less than 1 per cent with respect to regular CCSNe) pollutes its environment, the $r$-process elemental ratio is extremely high in comparison to regions where such a pollution did not take place. This inhomogeneity effect especially at low(est) metallicities might thus be an explanation for the observed large scatter in $r$-process elemental abundances. A key element to test the inhomogeneous halo issue is Eu. In particular, the ICE model suggests that different nucleosynthesis sources (neutron star mergers and fast rotating CCSNe driven by high magnetic fields) are needed to reproduce the large Eu spread observed in the Galactic halo (Wehmeyer et al. 2015).

We have seen in Section 2 that spectroscopic observations for the same star may differ from one analysis to the other for several reasons. In the same way, different results can be obtained also for theoretical GCE predictions. While for some elements (e.g. Mn), the theoretical GCE results are quite close to each other, for most of the elements large variations are obtained. In particular, the OMEGA prediction using the NUGRID yields (black dotted lines) show the largest departures from other models, and for many cases (e.g. Ni and $\mathrm{Zn}$ ) from the observations. The larger $[\mathrm{Co} / \mathrm{Fe}],[\mathrm{Ni} / \mathrm{Fe}],[\mathrm{Cu} / \mathrm{Fe}]$, $[\mathrm{Zn} / \mathrm{Fe}]$, and $[\mathrm{Zr} / \mathrm{Fe}]$ are due to the contribution from the $\alpha$-rich freezeout component (e.g. Woosley \& Hoffman 1992; Pignatari et al. 2016), present in CCSNe models from stellar progenitors with initial mass 12 and $15 \mathrm{M}_{\odot}$. The $[\mathrm{Cr} / \mathrm{Fe}]$ bump at solar-like metallicities is only due to the yields of the $20 \mathrm{M}_{\odot}$ star model that are affected by the merger of the $\mathrm{C}$ and $\mathrm{O}$ shells.

Taking into account the large variation between the different predictions, all the models cannot reproduce the observed trend for $[\mathrm{Sc} / \mathrm{Fe}],[\mathrm{Ti} / \mathrm{Fe}]$, and $[\mathrm{V} / \mathrm{Fe}]$. These issues are well known, and there is not yet a clear solution at least for TI and V (e.g. Kobayashi, Karakas \& Umeda 2011; Sneden et al. 2016). Concerning Sc, it was shown by Fröhlich et al. (2006b) that when neutrino interactions with the innermost ejected zones are treated correctly, Sc underabundance in the CCSN ejecta is strongly reduced. This is due to the effect that neutrinos increase the electron fraction Ye slightly above 0.5 . However, none of the presently existing yield tables for GCE studies take into account of these results. This issue could indicate that in real CCSNe, a range of entropies and electron fractions are obtained the ejected matter, which can be only obtained within multidimensional simulations. Therefore, for these cases, the presently available predictions of CCSN nucleosynthesis suffer the shortcomings that none of them are based on self-consistent multidimensional explosion models. This leads to three types of problems: (1) one-dimensional piston as well as thermal bomb methods utilize assumed explosion energies of the order $1-1.2 \times 10^{51} \mathrm{erg}$. This does not reflect differences in the pre-explosion stellar models, e.g. changing compactness, and therefore a variation in the expected range of explosion energies and mass cuts, related strongly to ${ }^{56} \mathrm{Ni}$ and other Fe-group ejecta. Hopefully more sophisticated upcoming approaches like PHOTB or PUSH can solve this issue (Perego et al. 2015; Sukhbold et al. 2016). (2) The presently utilized models do not include the effect of neutrino interactions with matter deep in explosive layers. Neutrino and antineutrino absorption on protons and neutrons leads to slightly proton-rich conditions, based on the neutron/proton mass difference for similar neutrino and antineutrino spectra and luminosities. This can improve the predictions for Sc, $\mathrm{Cu}$, and $\mathrm{Zn}$ (Fröhlich et al. 2006b,a). (3) In addition, limitations of 
one-dimensional CCSN models, neglecting the role of convection (which is the key aspect in realistic three-dimensional explosions) are affecting the robustness of nucleosynthesis results in particular for intermediate-mass and Fe-group elements. Most likely, these limitations are already relevant in the final stages of stellar progenitor models, and are also related to the difficulties in obtaining robust explosions from the last generation of CCSN multidimensional simulations (e.g. Müller 2016, and references therein).

For $\mathrm{Cr}, \mathrm{Mn}$, and $\mathrm{Ni}$, the predictions from the three-zone code (red lines) are similar to the ones of OMEGA (black solid and dashed lines). This better agreement compared to light $\alpha$ elements such as $\mathrm{Mg}$ implies that $\mathrm{Cr}, \mathrm{Mn}$, and $\mathrm{Ni}$ are not significantly affected by the ejecta of stars more massive than $30 \mathrm{M}_{\odot}$, as OMEGA did not include them. The large scatter seen for elements heavier than $\mathrm{Zn}$ at low $[\mathrm{Fe} / \mathrm{H}]$ between the GCE models is caused by the different assumptions used for the $r$-process and the $s$-process. Concerning the $r$-process elements (e.g. Eu), in the three-zone (red lines), GETOOL (green lines), and ICE (pink crosses) codes, some CCSNe contribute to the evolution and provide a short-time-scale enrichment that allows an early appearance of predictions on the $[\mathrm{Fe} / \mathrm{H}]$ axis. On the other hand, the OMEGA code (black lines) only considered the contribution of neutron star mergers, which require a certain delay before contributing to the chemical evolution of heavy elements. The ICE model also considered the contribution of neutron star mergers, but an additional earlier $r$-process source is included in the simulations (Wehmeyer et al. 2015). The variations seen between the black lines are only caused by different Fe yields associated with different choices of massive star models, as the same number of neutron star mergers and the same $r$-process yields were used. The choice of stellar yields can thus have an impact on the interpretation of how many $r$-process events is needed in order to explain the observations, at least when $\mathrm{Fe}$ is the element of reference in the abundance ratios. Concerning the prediction for typical $s$-process elements (e.g. Ba), the predictions by OMEGA are lower than observations. This is due to the adopted $s$-process yields from AGB models. For these simulations, the convective-boundary mixing mechanism assumed to be responsible for the formation of the ${ }^{13} \mathrm{C}$ pocket are producing a weaker $s$-process production compared to the most $s$-process rich AGB stars observed in the galactic disc, and compared to measurements in pre-solar mainstream $\mathrm{SiC}$ grains (Pignatari et al. 2016). These GCE results confirm these conclusions over the galactic metallicity range, and provide additional insights about physics processes relevant for stellar physics.

The ICE model (pink crosses), at least for $\mathrm{O}, \mathrm{Mg}, \mathrm{Si}, \mathrm{Ca}$, and $\mathrm{Eu}$, suggests that non-uniform mixing in the interstellar medium can generate scatter in the predictions that is larger than the scatter seen in the different components of the three-zone code, which means that data can be reproduced without implying the Galactic structure. On the other hand, the three-zone code can address the formation history of the different components of our Galaxy, which cannot be done with the ICE model. Secondly, using different stellar yields can also lead to large differences in the theoretical GCE simulations. For instance, it is still controversial the role of hypernovae to explain observations of iron-group elements (e.g. Nomoto et al. 2013; Sneden et al. 2016), while the impact of using different stellar yields is so relevant (see e.g. Romano et al. 2010; Mollá et al. 2015, and the discussion in this section). The controversial GCE role of different types of SNe Ia can also be considered as a similar source of uncertainty at $[\mathrm{Fe} / \mathrm{H}]$ above $\sim-1$ (e.g. Seitenzahl et al. 2013; Mishenina et al. 2015b). However, at present none of these scenarios have been established and a definitive solution for $\mathrm{Sc}, \mathrm{Ti}$, and V still needs to be found. For heavy elements, there are even more uncertainties to consider. The existence of a large variety of nucleosynthesis mechanisms highlighted from observations in the early Galaxy (e.g. Roederer et al. 2010; Hansen et al. 2012; Roederer et al. 2016; Frischknecht et al. 2016) in principle does not provide strong constraints about their effective relevance for the chemical inventory of the Sun (Travaglio et al. 2004; Bisterzo et al. 2014; Trippella et al. 2014; Cristallo et al. 2015). Nevertheless, there are strong observational indications now that the nucleosynthesis paradigm where the abundances beyond $\mathrm{Fe}$ are just made by a sum of $s$-process and $r$-process need to be revised (e.g. Mishenina et al. 2015a).

\section{CONCLUSIONS}

In this work, we presented and discussed the abundance measurements of 10 stars, with metallicity $-2.2<[\mathrm{Fe} / \mathrm{H}]<-0.6$. The same objects have been previously analysed by other authors, using different spectroscopic lines, methods, and assumptions. For most studied stars, the observed abundances for all the elements are consistent between all the authors. The largest discrepancy is obtained for star HD19445 for Al, whose abundance was determined with and without consideration of NLTE corrections. Some discrepancy for HD103095 between our determinations and Jofré et al. (2014) is due to the difference in temperature obtained in these two studies. And also, the discrepancy for the peculiar star HD6833 is possibly due to the small number of iron lines used to determine the metallicity by Fulbright (2000). In our case, the $\mathrm{O}$ abundance has the largest error, ranging between 0.10 and $0.2 \mathrm{dex}$, it is due to the $\mathrm{O}$ weak lines that we used. The best measured abundances are of $\mathrm{Cr}$, $\mathrm{Fe}$, and $\mathrm{Mn}$, the errors are between 0.03 and 0.11 dex.

We have compared the observations with an extended sample of predictions from GCE simulations. The study of the origin of the elements is based on the comparison between observations and GCE predictions. However, while stellar observations have usually provided with a clear error analysis, uncertainty in the theoretical GCE results need also to be considered. The main sources of this uncertainty are from stellar yields and from different assumptions in GCE simulations, among others, the stellar mass range on which stellar yields are applied, the interpolation scheme between stellar models, the stellar IMF, the star formation history, the star formation efficiency (related to the gas fraction), the treatment of $\mathrm{SNe}$ Ia, the astrophysical sites for heavy elements, and the galaxy framework (single or multizones). Different GCE models produce a scatter larger than observational errors for many elements. Furthermore, all these theoretical simulations are not consistent with the observed chemical evolution of the elemental ratios [Sc/Fe], [Ti/Fe], and $[\mathrm{V} / \mathrm{Fe}]$. While for Ti and $\mathrm{V}$, a clear solution has not been found yet, a promising scenario to solve the Sc problem has been discussed by Fröhlich et al. (2006b), but the impact of neutrino-winds ejecta still need to be tested within a GCE context. These problems not new, and here we can confirm them by using the results of four GCE codes. In particular, we considered six GCE models, including different theoretical stellar yields and a large variety of assumptions. We highlight that the present theoretical stellar yields from CCSNe are most likely the dominant source of this discrepancy between theory and observations, which is one of the most important puzzle that modern multidimensional CCSN simulations will need to solve. This underlines that improved CCSN nucleosynthesis predictions from realistic models are required. Among others, this requires a detailed study of the progenitor stellar structure in the last days before the $\mathrm{SN}$ explosion, the role of rotation and magnetic fields, the effect of neutrino interactions on the innermost ejected layers, and more 
substantially the role of the multidimensional explosion character. This and the possible role of hypernovae events for more massive stars are pre-requisites to a more comprehensive representation of the chemical evolution of the Galaxy.

\section{ACKNOWLEDGEMENTS}

We thank the anonymous referee for very helpful comments and suggestions. TM, TG, MP, FKT, and SK thank for the support from the Swiss National Science Foundation, project SCOPES no. IZ73Z0152485. MP acknowledges significant support to NUGRID from NSF grants PHY 09-22648 (Joint Institute for Nuclear Astrophysics, JINA), NSF grant PHY-1430152 (JINA Center for the Evolution of the Elements), and EU MIRG-CT-2006-046520. MP acknowledges the support from SNF (Switzerland). BC acknowledges financial support from the FRQNT (Quebec, Canada) postdoctoral fellowship program. FKT acknowledges support from the Swiss SNF and the European Research Council (FP7) under ERC Advanced Grant Agreement 321263 FISH. SB thanks JINA (ND Fund 202476) for financial support. BKG acknowledges the support of STFC, through grants ST/J001341/1 and ST/G003025/1. Numerical calculations have been supported by access to facilities, including the B2FH Association (http://www.b2fh.org/) and the University of Hull's High Performance Computing Facility, viper.

\section{REFERENCES}

Adibekyan V. Z., Sousa S. G., Santos N. C., Delgado Mena E., González Hernández J. I., Israelian G., Mayor M., Khachatryan G., 2012, A\&A, 545, A32

Allen C., Santillan A., 1993, Rev. Mex. Astron. Astrofis., 25, 39

Alonso A., Arribas S., Martinez-Roger C., 1996, A\&A, 313, 873

Alonso A., Arribas S., Martínez-Roger C., 1999, A\&AS, 140, 261

Andrievsky S. M., Spite M., Korotin S. A., Spite F., Bonifacio P., Cayrel R., Hill V., François P., 2008, A\&A, 481, 481

Andrievsky S. M., Spite M., Korotin S. A., Spite F., François P., Bonifacio P., Cayrel R., Hill V., 2009, A\&A, 494, 1083

Andrievsky S. M., Spite F., Korotin S. A., François P., Spite M., Bonifacio P., Cayrel R., Hill V., 2011, A\&A, 530, A105

Aoki W., Honda S., 2008, PASJ, 60, L7

Aoki W., Beers T. C., Christlieb N., Norris J. E., Ryan S. G., Tsangarides S., 2007, ApJ, 655, 492

Arcones A., Montes F., 2011, ApJ, 731, 5

Arcones A., Thielemann F.-K., 2013, J. Phys. G: Nucl. Phys., 40, 013201

Arlandini C., Käppeler F., Wisshak K., Gallino R., Lugaro M., Busso M., Straniero O., 1999, ApJ, 525, 886

Arnould M., Goriely S., Takahashi K., 2007, Phys. Rep., 450, 97

Asplund M., Lambert D. L., Nissen P. E., Primas F., Smith V. V., 2006, ApJ, 644, 229

Asplund M., Grevesse N., Sauval A. J., Scott P., 2009, ARA\&A, 47, 481

Battistini C., Bensby T., 2015, A\&A, 577, A9

Battistini C., Bensby T., 2016, A\&A, 586, A49

Bensby T., Feltzing S., Oey M. S., 2014, A\&A, 562, A71

Bergemann M., Gehren T., 2008, A\&A, 492, 823

Bergemann M., Lind K., Collet R., Magic Z., Asplund M., 2012, MNRAS, 427, 27

Bertolli M. G., Herwig F., Pignatari M., Kawano T., 2013, preprint (arXiv:1310.4578)

Biazzo K., D’Orazi V., Desidera S., Covino E., Alcalá J. M., Zusi M., 2012, MNRAS, 427, 2905

Bienaymé O., 1999, A\&A, 341, 86

Bisterzo S., Travaglio C., Gallino R., Wiescher M., Käppeler F., 2014, ApJ, 787, 10

Boesgaard A. M., Rich J. A., Levesque E. M., Bowler B. P., 2011, ApJ, 743, 140

Bonifacio P., Sbordone L., Caffau E., Ludwig H.-G., Spite M., González Hernández J. I., Behara N. T., 2012, A\&A, 542, A87
Bonifacio P. et al., 2015, A\&A, 579, A28

Bressan A., Marigo P., Girardi L., Salasnich B., Dal Cero C., Rubele S., Nanni A., 2012, MNRAS, 427, 127

Burbidge E. M., Burbidge G. R., Fowler W. A., Hoyle F., 1957, Rev. Mod. Phys., 29, 547

Burris D. L., Pilachowski C. A., Armandroff T. E., Sneden C., Cowan J. J., Roe H., 2000, ApJ, 544, 302

Carlsson M., 1986, Uppsala Astronomical Observatory Reports, 33, A Computer Program for Solving Multi-Level Non-LTE Radiative Transfer Problems in Moving or Static Atmospheres. Uppsala Astronomical Observatory, Uppsala, Sweden

Carollo D. et al., 2010, ApJ, 712, 692

Carraro G., 2015, IAU General Assembly Meeting, 22, 2254036

Casagrande L., Ramírez I., Meléndez J., Bessell M., Asplund M., 2010, A\&A, 512, A54

Casagrande L., Schönrich R., Asplund M., Cassisi S., Ramírez I., Meléndez J., Bensby T., Feltzing S., 2011, A\&A, 530, A138

Castelli F., Kurucz R. L., 2004, preprints (arXiv:astro-ph/0405087)

Cayrel R., Spite M., Spite F., Vangioni-Flam E., Cassé M., Audouze J., 1999, A\&A, 343, 923

Chen Y. Q., Nissen P. E., Zhao G., Zhang H. W., Benoni T., 2000, A\&AS, 141,491

Chiappini C., Hirschi R., Meynet G., Ekström S., Maeder A., Matteucci F., 2006, A\&A, 449, L27

Chieffi A., Limongi M., 2013, ApJ, 764, 21

Cho D.-H., Sung H.-I., Lee S.-G., Yoon T. S., 2016, J. Korean Astron. Soc., 49,175

Cohen J. G., Christlieb N., Thompson I., McWilliam A., Shectman S., Reimers D., Wisotzki L., Kirby E., 2013, ApJ, 778, 56

Colucci J. E., Bernstein R. A., Cameron S. A., McWilliam A., 2012, ApJ, 746, 29

Côté B., West C., Heger A., Ritter C., O'Shea B. W., Herwig F., Travaglio C., Bisterzo S., 2016a, MNRAS, 463, 3755

Côté B., Ritter C., O'Shea B. W., Herwig F., Pignatari M., Jones S., Fryer C. L., 2016b, ApJ, 824, 82

Côté B., O’Shea B. W., Ritter C., Herwig F., Venn K. A., 2017, ApJ, 835 , 128

Cowan J. J., Rose W. K., 1977, ApJ, 212, 149

Cristallo S., Abia C., Straniero O., Piersanti L., 2015, ApJ, 801, 53

Cyburt R. H., Fields B. D., Olive K. A., 2008, J. Cosmol. Astropart. Phys., 11,012

Cyburt R. H., Fields B. D., Olive K. A., Yeh T.-H., 2016, Rev. Mod. Phys., 88,015004

Dardelet L. et al., 2014, Proc. Sci., The i-process and CEMP-r/s stars. SISSA, Trieste. PoS\#145

Delgado Mena E. et al., 2014, A\&A, 562, A92

Den Hartog E. A., Lawler J. E., Sneden C., Cowan J. J., 2006, ApJS, 167, 292

Denissenkov P. A., Merryfield W. J., 2011, ApJ, 727, L8

Denissenkov P. A., Weiss A., 1996, A\&A, 308, 773

Denissenkov P. A., Da Costa G. S., Norris J. E., Weiss A., 1998, A\&A, 333, 926

Denissenkov P. A., VandenBerg D. A., Hartwick F. D. A., Herwig F., Weiss A., Paxton B., 2015, MNRAS, 448, 3314

Dominik M., Belczynski K., Fryer C., Holz D. E., Berti E., Bulik T., Mandel I., O'Shaughnessy R., 2012, ApJ, 759, 52

Ertl T., Janka H.-T., Woosley S. E., Sukhbold T., Ugliano M., 2016, ApJ, 818,124

Farouqi K., Kratz K., Mashonkina L. I., Pfeiffer B., Cowan J. J., Thielemann F., Truran J. W., 2009, ApJ, 694, L49

Fenner Y., Gibson B. K., 2003, PASA, 20, 189

Fields B. D., 2011, Annu. Rev. Nucl. Part. Sci., 61, 47

Flower P. J., 1975, A\&A, 41, 391

Frebel A., 2010, Astron. Nachr., 331, 474

Frischknecht U. et al., 2016, MNRAS, 456, 1803

Fröhlich C., Martínez-Pinedo G., Liebendörfer M., Thielemann F.-K., Bravo E., Hix W. R., Langanke K., Zinner N. T., 2006a, Phys. Rev. Lett., 96, 142502

Fröhlich C. et al., 2006b, ApJ, 637, 415 
Fryer C. L., Belczynski K., Wiktorowicz G., Dominik M., Kalogera V., Holz D. E., 2012, ApJ, 749, 91

Fulbright J. P., 2000, AJ, 120, 1841

Gaia Collaboration et al., 2016, A\&A, 595, A2

Galazutdinov G. A., 1992, preprint (arXiv:e-prints)

Gibson B. K., 1997, MNRAS, 290, 471

Gibson B. K., Fenner Y., Renda A., Kawata D., Lee H.-c., 2003, PASA, 20, 401

Girardi L., Bertelli G., Bressan A., Chiosi C., Groenewegen M. A. T., Marigo P., Salasnich B., Weiss A., 2002, A\&A, 391, 195

González Hernández J. I., Bonifacio P., 2009, A\&A, 497, 497

Goriely S., 2008, in Demetriou P., Julin R., Harissopulos S. V., eds, AIP Conf. Ser. Vol. 1012, Frontiers in Nuclear Structure, Astrophysics, and Reactions: Finustar 2. Am. Inst. Phys., New York, pp. 38-45.

Gratton R. G., Sneden C., Carretta E., Bragaglia A., 2000, A\&A, 354, 169

Gratton R. G., Carretta E., Claudi R., Lucatello S., Barbieri M., 2003, A\&A, 404, 187

Gray R. O., Corbally C. J., Garrison R. F., McFadden M. T., Robinson P. E., 2003, AJ, 126, 2048

Griffen B. F., Dooley G. A., Ji A. P., O'Shea B. W., Gómez F. A., Frebel A., 2016, MNRAS, preprint (arXiv:1611.00759)

Guiglion G., de Laverny P., Recio-Blanco A., Worley C. C., De Pascale M., Masseron T., Prantzos N., Mikolaitis Š., 2016, A\&A, 595, A18

Hampel M., Stancliffe R. J., Lugaro M., Meyer B. S., 2016, ApJ, 831, 171

Hansen C. J., Primas F., 2011, A\&A, 525, L5

Hansen C. J. et al., 2012, A\&A, 545, A31

Hansen C. J., Bergemann M., Cescutti G., François P., Arcones A., Karakas A. I., Lind K., Chiappini C., 2013, A\&A, 551, A57

Hawkins K., Jofré P., Masseron T., Gilmore G., 2015, MNRAS, 453, 758

Heiter U., Jofré P., Gustafsson B., Korn A. J., Soubiran C., Thévenin F., 2015, A\&A, 582, A49

Herwig F., Pignatari M., Woodward P. R., Porter D. H., Rockefeller G., Fryer C. L., Bennett M., Hirschi R., 2011, ApJ, 727, 89

Hillebrandt W., Kromer M., Röpke F. K., Ruiter A. J., 2013, Front. Phys., 8,116

Hinkel N. R., Timmes F. X., Young P. A., Pagano M. D., Turnbull M. C., 2014, AJ, 148, 54

Hobbs L. M., Thorburn J. A., 1994, ApJ, 428, L25

Holmberg J., Nordström B., Andersen J., 2009, A\&A, 501, 941

Hughes G. L., Gibson B. K., Carigi L., Sánchez-Blázquez P., Chavez J. M., Lambert D. L., 2008, MNRAS, 390, 1710

Ishigaki M. N., Chiba M., Aoki W., 2012, ApJ, 753, 64

Ishigaki M. N., Aoki W., Chiba M., 2013, ApJ, 771, 67

Ishigaki M. N., Aoki W., Arimoto N., Okamoto S., 2014, A\&A, 562, A146

Ivans I. I., Simmerer J., Sneden C., Lawler J. E., Cowan J. J., Gallino R., Bisterzo S., 2006, ApJ, 645, 613

Iwamoto K., Brachwitz F., Nomoto K., Kishimoto N., Umeda H., Hix W. R., Thielemann F.-K., 1999, ApJS, 125, 439

Jofré P. et al., 2014, A\&A, 564, A133

Jofré P. et al., 2015, A\&A, 582, A81

Jones S., Hirschi R., Pignatari M., Heger A., Georgy C., Nishimura N., Fryer C., Herwig F., 2015, MNRAS, 447, 3115

Käppeler F., Gallino R., Bisterzo S., Aoki W., 2011, Rev. Mod. Phys., 83, 157

Karakas A., Lattanzio J. C., 2007, PASA, 24, 103

Klochkova V. G., Mishenina T. V., Panchuk V. E., Korotin S. A., Marsakov V. A., Usenko I. A., Tsymbal V. V., 2011, Astrophys. Bull., 66, 28

Kobayashi C., Karakas A. I., Umeda H., 2011, MNRAS, 414, 3231

Korobkin O., Rosswog S., Arcones A., Winteler C., 2012, MNRAS, 426, 1940

Korotin S. A., Andrievsky S. M., Luck R. E., 1999, A\&A, 351, 168

Kroupa P., Tout C. A., Gilmore G., 1993, MNRAS, 262, 545

Kupka F., Piskunov N., Ryabchikova T. A., Stempels H. C., Weiss W. W., 1999, A\&AS, 138, 119

Kurucz R., 1993, ATLAS9 Stellar Atmosphere Programs and $2 \mathrm{~km} / \mathrm{s}$ Grid. Kurucz CD-ROM No. 13. Smithsonian Astrophysical Observatory, Cambridge, MA

Lai D. K., Bolte M., Johnson J. A., Lucatello S., Heger A., Woosley S. E., 2008, ApJ, 681, 1524
Lattanzio J., Forestini M., 1999, in Bertre T. L., Lebre A., Waelkens C., eds, IAU Symp. 191. AGB Stars. Astron. Soc. Pac., San Francisco, p. 31

Lattanzio J. C., Siess L., Church R. P., Angelou G., Stancliffe R. J., Doherty C. L., Stephen T., Campbell S. W., 2015, MNRAS, 446, 2673

Lawler J. E., Den Hartog E. A., Sneden C., Cowan J. J., 2006, ApJS, 162, 227

Lawler J. E., Sneden C., Cowan J. J., Ivans I. I., Den Hartog E. A., 2009, ApJS, 182, 51

Lawler J. E., Guzman A., Wood M. P., Sneden C., Cowan J. J., 2013, ApJS, 205,11

Limongi M., Chieffi A., 2012, ApJS, 199, 38

Lind K., Melendez J., Asplund M., Collet R., Magic Z., 2013, A\&A, 554, A96

Livshits M. A., 1997, Sol. Phys., 173, 377

Luck R. E., 1991, ApJS, 75, 579

Maldonado J., Eiroa C., Villaver E., Montesinos B., Mora A., 2012, A\&A, 541, A40

Maoz D., Mannucci F., Nelemans G., 2014, ARA\&A, 52, 107

Mashonkina L., Gehren T., 2000, A\&A, 364, 249

Mashonkina L., Gehren T., Shi J.-R., Korn A. J., Grupp F., 2011, A\&A, 528, A87

Mashonkina L., Ryabtsev A., Frebel A., 2012, A\&A, 540, A98

Matteucci F., Tornambe A., 1985, A\&A, 142, 13

Meléndez J., Casagrande L., Ramírez I., Asplund M., Schuster W. J., 2010, A\&A, 515, L3

Mishenina T. V., Kovtyukh V. V., 2001, A\&A, 370, 951

Mishenina T. V., Tsymbal V. V., 1997, Astron. Lett., 23, 609

Mishenina T. V., Soubiran C., Kovtyukh V. V., Korotin S. A., 2004, A\&A, 418, 551

Mishenina T. V., Gorbaneva T. I., Basak N. Y., Soubiran C., Kovtyukh V. V., 2011, Astron. Rep., 55, 689

Mishenina T. et al., 2015a, MNRAS, 446, 3651

Mishenina T., Gorbaneva T., Pignatari M., Thielemann F.-K., Korotin S. A., 2015b, MNRAS, 454, 1585

Molenda-Żakowicz J. et al., 2013, MNRAS, 434, 1422

Mollá M., Cavichia O., Gavilán M., Gibson B. K., 2015, MNRAS, 451, 3693

Müller B., 2016, PASA, 33, e048

Nishimura N., Takiwaki T., Thielemann F.-K., 2015, ApJ, 810, 109

Nissen P. E., Schuster W. J., 2010, A\&A, 511, L10

Nissen P. E., Schuster W. J., 2011, A\&A, 530, A15

Nissen P. E., Chen Y. Q., Schuster W. J., Zhao G., 2000, A\&A, 353, 722

Nollett K. M., Busso M., Wasserburg G. J., 2003, ApJ, 582, 1036

Nomoto K., Iwamoto K., Nakasato N., Thielemann F.-K., Brachwitz F., Tsujimoto T., Kubo Y., Kishimoto N., 1997, Nucl. Phys. A, 621, 467

Nomoto K., Kobayashi C., Tominaga N., 2013, ARA\&A, 51, 457

Palmerini S., La Cognata M., Cristallo S., Busso M., 2011, ApJ, 729, 3

Perego A., Hempel M., Fröhlich C., Ebinger K., Eichler M., Casanova J., Liebendörfer M., Thielemann F.-K., 2015, ApJ, 806, 275

Perruchot S. et al., 2008, in McLean I. S., Casali M. M., eds, Proc. SPIE Conf. Ser. Vol. 7014, Ground-based and Airborne Instrumentation for Astronomy II. SPIE, Bellingham, p. 12

Pignatari M. et al., 2015, ApJ, 808, L43

Pignatari M. et al., 2016, ApJS, 225, 24

Planck Collaboration I, 2016, A\&A, 594, A1

Prantzos N., 2012, A\&A, 542, A67

Prantzos N., Charbonnel C., Iliadis C., 2007, A\&A, 470, 179

Prochaska J. X., McWilliam A., 2000, ApJ, 537, L57

Qian Y.-Z., Wasserburg G. J., 2008, ApJ, 687, 272

Ramírez I., Allende Prieto C., Lambert D. L., 2007, A\&A, 465, 271

Ramírez I., Fish J. R., Lambert D. L., Allende Prieto C., 2012, ApJ, 756, 46

Ramírez I., Allende Prieto C., Lambert D. L., 2013, ApJ, 764, 78

Reddy B. E., Tomkin J., Lambert D. L., Allende Prieto C., 2003, MNRAS, 340, 304

Reddy B. E., Lambert D. L., Allende Prieto C., 2006, MNRAS, 367, 1329

Roberts L. F., Woosley S. E., Hoffman R. D., 2010, ApJ, 722, 954

Roederer I. U., Kratz K.-L., Frebel A., Christlieb N., Pfeiffer B., Cowan J. J., Sneden C., 2009, ApJ, 698, 1963 
Roederer I. U., Cowan J. J., Karakas A. I., Kratz K.-L., Lugaro M., Simmerer J., Farouqi K., Sneden C., 2010, ApJ, 724, 975

Roederer I. U., Preston G. W., Thompson I. B., Shectman S. A., Sneden C., Burley G. S., Kelson D. D., 2014a, AJ, 147, 136

Roederer I. U., Preston G. W., Thompson I. B., Shectman S. A., Sneden C., Burley G. S., Kelson D. D., 2014b, AJ, 147, 136

Roederer I. U., Karakas A. I., Pignatari M., Herwig F., 2016, ApJ, 821, 37

Romano D., Karakas A. I., Tosi M., Matteucci F., 2010, A\&A, 522, A32

Rutten R. J., 1978, Sol. Phys., 56, 237

Sackmann I. J., Boothroyd A. I., 1999, ApJ, 510, 217

Schaller G., Schaerer D., Meynet G., Maeder A., 1992, A\&AS, 96, 269

Seitenzahl I. R., Cescutti G., Röpke F. K., Ruiter A. J., Pakmor R., 2013, A\&A, 559, L5

Simmerer J., Sneden C., Cowan J. J., Collier J., Woolf V. M., Lawler J. E., 2004, ApJ, 617, 1091

Sitnova T. et al., 2015, ApJ, 808, 148

Smith V. V., Lambert D. L., Nissen P. E., 1993, ApJ, 408, 262

Sneden C., Cowan J. J., Gallino R., 2008, ARA\&A, 46, 241

Sneden C., Lawler J. E., Cowan J. J., Ivans I. I., Den Hartog E. A., 2009, ApJS, 182, 80

Sneden C., Cowan J. J., Kobayashi C., Pignatari M., Lawler J. E., Den Hartog E. A., Wood M. P., 2016, ApJ, 817, 53

Soubiran C., Bienaymé O., Siebert A., 2003, A\&A, 398, 141

Sousa S. G. et al., 2008, A\&A, 487, 373

Sozzetti A., Torres G., Latham D. W., Stefanik R. P., Korzennik S. G., Boss A. P., Carney B. W., Laird J. B., 2009, ApJ, 697, 544

Spergel D. N. et al., 2003, ApJS, 148, 175

Spite F., Spite M., 1982, A\&A, 115, 357

Spite M. et al., 2005, A\&A, 430, 655

Spite M. et al., 2012, A\&A, 541, A143

Steigman G., 2007, Annu. Rev. Nucl. Part. Sci., 57, 463

Stonkute E., Tautvaišiene G., Nordström B., Ženoviene R., 2012, A\&A, 541, A157

Sukhbold T., Ertl T., Woosley S. E., Brown J. M., Janka H.-T., 2016, ApJ, 821,38

Takeda Y., 2007, PASJ, 59, 335

Takeda Y., Ohkubo M., Sato B., Kambe E., Sadakane K., 2005, PASJ, 57, 27

Tegmark M. et al., 2004, Phys. Rev. D, 69, 103501

Thielemann F.-K., Nomoto K., Yokoi K., 1986, A\&A, 158, 17

Thielemann F.-K., Nomoto K., Hashimoto M.-A., 1996, ApJ, 460, 408

Thielemann F.-K. et al., 2011a, Prog. Part. Nucl. Phys., 66, 346

Thielemann F.-K., Hirschi R., Liebendörfer M., Diehl R., 2011b, in Diehl R., Hartmann D. H., Prantzos N., eds, Lecture Notes in Physics, Vol. 812, Astronomy with Radioactivities. Springer-Verlag, Berlin, p. 153

Thorburn J. A., Hobbs L. M., Deliyannis C. P., Pinsonneault M. H., 1993, ApJ, 415, 150

Travaglio C., Galli D., Gallino R., Busso M., Ferrini F., Straniero O., 1999, ApJ, 521, 691

Travaglio C., Gallino R., Arnone E., Cowan J., Jordan F., Sneden C., 2004, ApJ, 601, 864

Travaglio C., Hillebrandt W., Reinecke M., 2005, A\&A, 443, 1007

Trippella O., Busso M., Maiorca E., Käppeler F., Palmerini S., 2014, ApJ, 787, 41

Tsantaki M., Sousa S. G., Adibekyan V. Z., Santos N. C., Mortier A., Israelian G., 2013, A\&A, 555, A150

Tsymbal V., 1996, in Adelman S. J., Kupka F., Weiss W. W., eds, ASP Conf. Ser. Vol. 108, Model Atmospheres and Spectrum Synthesis. Astron. Soc. Pac., San Francisco, p. 198

van Leeuwen F. ed. 2007, in Astrophysics and Space Science Library, Vol. 350, Hipparcos, the New Reduction of the Raw Data. Springer-Verlag, Berlin

VandenBerg D. A., Bond H. E., Nelan E. P., Nissen P. E., Schaefer G. H., Harmer D., 2014, ApJ, 792, 110

Venn K. A., Irwin M., Shetrone M. D., Tout C. A., Hill V., Tolstoy E., 2004, AJ, 128, 1177

Wanajo S., Janka H.-T., Kubono S., 2011, ApJ, 729, 46

Wehmeyer B., Pignatari M., Thielemann F.-K., 2015, MNRAS, 452, 1970
Winteler C., Käppeli R., Perego A., Arcones A., Vasset N., Nishimura N., Liebendörfer M., Thielemann F.-K., 2012, ApJ, 750, L22

Woosley S. E., Hoffman R. D., 1992, ApJ, 395, 202

Woosley S. E., Weaver T. A., 1995, ApJS, 101, 181

Woosley S. E., Heger A., Weaver T. A., 2002, Rev. Mod. Phys., 74, 1015

Yan H. L., Shi J. R., Zhao G., 2015, ApJ, 802, 36

Yong D. et al., 2013, ApJ, 762, 26

Yoon J., Beers T. C., Placco V. M., Rasmussen K. C., Carollo D., He S., Hansen T. T., Roederer I. U., 2016, ApJ, 833, 20

Yushchenko A. et al., 2005, A\&A, 430, 255

Zhao G., Gehren T., 2000, A\&A, 362, 1077

\section{SUPPORTING INFORMATION}

Supplementary data are available at MNRAS online.

Table 7. Atomic data and EWs of used lines.

Please note: Oxford University Press is not responsible for the content or functionality of any supporting materials supplied by the authors. Any queries (other than missing material) should be directed to the corresponding author for the article.

\section{APPENDIX}

Table A1. Atomic data for the lines used in synthetic method calculations. NLTE calculations marked in table.

\begin{tabular}{|c|c|c|c|c|}
\hline $\mathrm{El}$ & $\begin{array}{c}\lambda \\
0.1 \mathrm{~nm}\end{array}$ & $\log g f$ & $\begin{array}{l}E_{\text {low }} \\
(\mathrm{eV})\end{array}$ & Note \\
\hline $\mathrm{O}_{\mathrm{I}}$ & 6300.304 & -9.717 & 0.000 & NLTE \\
\hline $\mathrm{Na} \mathrm{I}$ & 5682.630 & -0.708 & 2.102 & NLTE \\
\hline $\mathrm{Na} I$ & 5688.190 & -1.407 & 2.104 & NLTE \\
\hline $\mathrm{Na} \mathrm{I}$ & 5688.200 & -0.452 & 2.104 & NLTE \\
\hline $\mathrm{Na} \mathrm{I}$ & 5889.940 & 0.108 & 0.000 & NLTE \\
\hline $\mathrm{Na} I$ & 5895.920 & -0.195 & 0.000 & NLTE \\
\hline $\mathrm{Na} \mathrm{I}$ & 6154.220 & -1.560 & 2.102 & NLTE \\
\hline $\mathrm{Na} \mathrm{I}$ & 6160.740 & -1.260 & 2.104 & NLTE \\
\hline $\mathrm{Mg}_{\mathrm{I}}$ & 4167.271 & -0.745 & 4.346 & NLTE \\
\hline $\mathrm{Mg}_{\mathrm{I}}$ & 4702.990 & -0.440 & 4.346 & NLTE \\
\hline $\mathrm{Mg}_{\mathrm{I}}$ & 4730.020 & -2.292 & 4.346 & NLTE \\
\hline $\mathrm{Mg}_{\mathrm{I}}$ & 5172.680 & -0.451 & 2.712 & NLTE \\
\hline $\mathrm{Mg}_{\mathrm{I}}$ & 5183.600 & -0.240 & 2.717 & NLTE \\
\hline $\mathrm{Mg}_{\mathrm{I}}$ & 5528.400 & -0.498 & 4.346 & NLTE \\
\hline $\mathrm{Mg}_{\mathrm{I}}$ & 5711.080 & -1.720 & 4.346 & NLTE \\
\hline $\mathrm{Mg}_{\mathrm{I}}$ & 6318.710 & -1.839 & 5.108 & NLTE \\
\hline $\mathrm{Mg}_{\mathrm{I}}$ & 6319.230 & -2.060 & 5.108 & NLTE \\
\hline $\mathrm{Mg}_{\mathrm{I}}$ & 6319.490 & -2.537 & 5.108 & NLTE \\
\hline $\mathrm{Al}$ I & 3944.006 & -0.622 & 0.000 & NLTE \\
\hline $\mathrm{Al}$ I & 3961.520 & -0.322 & 0.014 & NLTE \\
\hline $\mathrm{Al}$ I & 5557.063 & -2.377 & 3.143 & NLTE \\
\hline $\mathrm{Al}$ I & 6696.023 & -1.479 & 3.143 & NLTE \\
\hline $\mathrm{Al}$ I & 6698.673 & -1.780 & 3.143 & NLTE \\
\hline $\mathrm{Ca} \mathrm{I}$ & 4108.526 & -0.824 & 2.709 & NLTE \\
\hline $\mathrm{Ca} \mathrm{I}$ & 4226.728 & 0.244 & 0.000 & NLTE \\
\hline $\mathrm{Ca} \mathrm{I}$ & 4283.011 & -0.220 & 1.886 & NLTE \\
\hline $\mathrm{Ca} \mathrm{I}$ & 4289.367 & -0.300 & 1.879 & NLTE \\
\hline $\mathrm{Ca} \mathrm{I}$ & 4302.528 & 0.280 & 1.899 & NLTE \\
\hline $\mathrm{Ca} \mathrm{I}$ & 4318.652 & -0.211 & 1.899 & NLTE \\
\hline $\mathrm{Ca} \mathrm{I}$ & 4355.079 & -0.420 & 2.709 & NLTE \\
\hline $\mathrm{Ca} \mathrm{I}$ & 4425.437 & -0.360 & 1.879 & NLTE \\
\hline $\mathrm{Ca} \mathrm{I}$ & 4434.957 & -0.010 & 1.886 & NLTE \\
\hline $\mathrm{Ca} \mathrm{I}$ & 4435.679 & -0.523 & 1.886 & NLTE \\
\hline $\mathrm{Ca} \mathrm{I}$ & 4454.779 & 0.260 & 1.899 & NLTE \\
\hline
\end{tabular}


Table A1 - continued

\begin{tabular}{|c|c|c|c|}
\hline El & $\begin{array}{c}\lambda \\
0.1 \mathrm{~nm}\end{array}$ & $\log g f$ & $\begin{array}{l}E_{\text {low }} \\
(\mathrm{eV})\end{array}$ \\
\hline $\mathrm{Ca} \mathrm{I}$ & 4512.268 & -1.892 & 2.526 \\
\hline $\mathrm{Ca} \mathrm{I}$ & 4526.928 & -0.548 & 2.709 \\
\hline $\mathrm{Ca} \mathrm{I}$ & 4578.551 & -0.697 & 2.521 \\
\hline $\mathrm{Ca} \mathrm{I}$ & 4685.268 & -0.880 & 2.933 \\
\hline $\mathrm{Ca} \mathrm{I}$ & 5188.844 & -0.115 & 2.933 \\
\hline $\mathrm{Ca}_{\mathrm{I}}$ & 5260.387 & -1.719 & 2.521 \\
\hline $\mathrm{Ca} \mathrm{I}$ & 5261.704 & -0.579 & 2.521 \\
\hline $\mathrm{Ca}$ I & 5265.556 & -0.114 & 2.523 \\
\hline $\mathrm{Ca} \mathrm{I}$ & 5349.465 & -0.310 & 2.709 \\
\hline $\mathrm{Ca} \mathrm{I}$ & 5512.980 & -0.464 & 2.933 \\
\hline $\mathrm{Ca}$ I & 5581.965 & -0.555 & 2.523 \\
\hline $\mathrm{Ca}$ I & 5588.749 & 0.358 & 2.526 \\
\hline $\mathrm{Ca} I$ & 5590.114 & -0.571 & 2.521 \\
\hline $\mathrm{Ca} I$ & 5594.462 & 0.097 & 2.523 \\
\hline $\mathrm{Ca}$ I & 5601.277 & -0.523 & 2.526 \\
\hline $\mathrm{Ca} \mathrm{I}$ & 5857.451 & 0.240 & 2.933 \\
\hline $\mathrm{Ca}$ I & 5867.562 & -1.570 & 2.933 \\
\hline $\mathrm{Ca}$ I & 6102.723 & -0.770 & 1.879 \\
\hline $\mathrm{Ca}_{\mathrm{I}}$ & 6122.217 & -0.319 & 1.886 \\
\hline $\mathrm{Ca} \mathrm{I}$ & 6162.173 & -0.090 & 1.899 \\
\hline $\mathrm{Ca} \mathrm{I}$ & 6163.755 & -1.286 & 2.521 \\
\hline $\mathrm{Ca} \mathrm{I}$ & 6166.439 & -1.143 & 2.521 \\
\hline $\mathrm{Ca}$ I & 6169.042 & -0.797 & 2.523 \\
\hline $\mathrm{Ca} \mathrm{I}$ & 6169.563 & -0.478 & 2.526 \\
\hline $\mathrm{Ca} \mathrm{I}$ & 6439.075 & 0.390 & 2.526 \\
\hline $\mathrm{Ca} I$ & 6462.567 & 0.262 & 2.523 \\
\hline $\mathrm{Ca}$ I & 6471.662 & -0.686 & 2.526 \\
\hline $\mathrm{Ca} \mathrm{I}$ & 6493.781 & -0.109 & 2.521 \\
\hline $\mathrm{Ca} \mathrm{I}$ & 6499.650 & -0.818 & 2.523 \\
\hline $\mathrm{Ca} \mathrm{I}$ & 6572.779 & -4.296 & 0.000 \\
\hline $\mathrm{Ca} \mathrm{I}$ & 6717.681 & -0.523 & 2.709 \\
\hline $\mathrm{Sc}$ II & 4670.400 & -0.580 & 1.357 \\
\hline $\mathrm{Sc}_{\text {II }}$ & 5239.823 & -0.770 & 1.455 \\
\hline $\mathrm{Sc}$ II & 5318.336 & -2.040 & 1.357 \\
\hline Sc II & 5526.770 & 0.130 & 1.768 \\
\hline $\mathrm{Sc}$ II & 5657.886 & -0.500 & 1.507 \\
\hline $\mathrm{Sc}_{\text {II }}$ & 5667.000 & -1.240 & 1.500 \\
\hline $\mathrm{Sc}_{\text {II }}$ & 5669.038 & -1.120 & 1.500 \\
\hline $\mathrm{Sc}$ II & 5684.190 & -1.080 & 1.507 \\
\hline $\mathrm{Sc}_{\text {II }}$ & 6245.621 & -0.980 & 1.507 \\
\hline $\mathrm{Sc}_{\text {II }}$ & 6604.582 & -1.480 & 1.357 \\
\hline $\mathrm{Mn}$ I & 4502.221 & -0.345 & 2.920 \\
\hline Mn I & 4709.720 & -0.340 & 2.890 \\
\hline Mn I & 4739.113 & -0.490 & 2.941 \\
\hline Mn I & 4754.039 & -0.086 & 2.282 \\
\hline Mn I & 4761.530 & -0.138 & 2.953 \\
\hline $\mathrm{Mn}_{\mathrm{I}}$ & 4762.375 & 0.425 & 2.889 \\
\hline Mn I & 4783.420 & 0.042 & 2.300 \\
\hline Mn I & 4823.514 & 0.144 & 2.320 \\
\hline Mn I & 5432.550 & -3.795 & 0.000 \\
\hline Mn I & 6013.497 & -0.251 & 3.073 \\
\hline Mn I & 6021.803 & 0.034 & 3.075 \\
\hline $\mathrm{CuI}$ & 5105.545 & 1.390 & -1.510 \\
\hline $\mathrm{CuI}$ & 5218.209 & 3.820 & 0.270 \\
\hline $\mathrm{CuI}$ & 5782.136 & 1.640 & -1.780 \\
\hline $\mathrm{Sr}$ II & 4077.709 & 0.167 & 0.000 \\
\hline $\mathrm{Sr}$ II & 4161.792 & -0.501 & 2.940 \\
\hline Sr II & 4215.519 & -0.144 & 0.000 \\
\hline $\mathrm{Y}_{\mathrm{II}}$ & 4374.933 & 0.271 & 0.409 \\
\hline $\mathrm{Y}_{\text {II }}$ & 4398.010 & -0.894 & 0.130 \\
\hline $\mathrm{Y}_{\text {II }}$ & 4854.876 & -0.110 & 0.990 \\
\hline $\mathrm{Y}_{\mathrm{II}}$ & 4883.682 & 0.265 & 1.080 \\
\hline $\mathrm{Y}_{\mathrm{II}}$ & 4900.119 & 0.103 & 1.033 \\
\hline
\end{tabular}

Table A1 - continued

\begin{tabular}{|c|c|c|c|c|}
\hline $\mathrm{El}$ & $\begin{array}{c}\lambda \\
0.1 \mathrm{~nm}\end{array}$ & $\log g f$ & $\begin{array}{l}E_{\text {low }} \\
(\mathrm{eV})\end{array}$ & Note \\
\hline$Y_{\text {II }}$ & 4982.129 & -1.289 & 1.033 & \\
\hline $\mathrm{Y}_{\text {II }}$ & 5087.416 & -0.169 & 1.084 & \\
\hline Y II & 5119.112 & -1.359 & 0.992 & \\
\hline Y II & 5200.406 & -0.569 & 0.992 & \\
\hline $\mathrm{Y}_{\text {II }}$ & 5205.722 & -0.192 & 1.033 & \\
\hline Y II & 5402.774 & -0.629 & 1.839 & \\
\hline Ba II & 4554.034 & 0.163 & 0.000 & HFS, NLTE \\
\hline Ba II & 5853.675 & -1.000 & 0.604 & HFS, NLTE \\
\hline $\mathrm{Ba}$ II & 6141.714 & -0.076 & 0.704 & HFS, NLTE \\
\hline Ba II & 6496.900 & -0.377 & 0.604 & HFS, NLTE \\
\hline La II & 4086.710 & -0.069 & 0.000 & \\
\hline La II & 4123.236 & 0.110 & 0.321 & \\
\hline La II & 4238.391 & -0.219 & 0.403 & \\
\hline La II & 4526.097 & -0.649 & 0.772 & \\
\hline La II & 4558.460 & -0.969 & 0.321 & \\
\hline La II & 4662.509 & -1.239 & 0.000 & \\
\hline La II & 4716.440 & -1.209 & 0.772 & \\
\hline La II & 4748.720 & -0.539 & 0.927 & \\
\hline La II & 4921.790 & -0.449 & 0.244 & \\
\hline La II & 4986.830 & -1.299 & 0.173 & \\
\hline La II & 5122.989 & -0.849 & 0.321 & \\
\hline La II & 5163.612 & -1.809 & 0.244 & \\
\hline La II & 5290.840 & -1.649 & 0.000 & \\
\hline La II & 5303.530 & -1.349 & 0.321 & \\
\hline La II & 5482.270 & -2.229 & 0.000 & \\
\hline La II & 5808.310 & -2.199 & 0.000 & \\
\hline La II & 6390.480 & -1.409 & 0.321 & \\
\hline La II & 6774.000 & -1.819 & 0.126 & \\
\hline Pr II & 4222.950 & 0.235 & 0.055 & HFS \\
\hline $\operatorname{Pr}$ II & 4408.820 & 0.053 & 0.000 & HFS \\
\hline Pr II & 4510.150 & -0.007 & 0.422 & HFS \\
\hline Pr II & 5259.730 & 0.114 & 0.633 & HFS \\
\hline Pr II & 5322.770 & -0.123 & 0.482 & HFS \\
\hline
\end{tabular}

4018.810

Nd II $\quad 4021.340$

Nd II $\quad 4069.260$

Nd II $\quad 4368.630$

Nd II $\quad 4446.380$

Nd II $\quad 4462.980$

Nd II $\quad 4501.810$

Nd II $\quad 4706.540$

4797.150

4825.480

$-0.849$

0.064

$\begin{array}{ll}-0.099 & 0.321\end{array}$

$\begin{array}{ll}-0.390 & 0.064\end{array}$

$\begin{array}{ll}-0.809 & 0.064\end{array}$

$-0.349 \quad 0.205$

$0.040 \quad 0.559$

$\begin{array}{ll}-0.689 & 0.205\end{array}$

$\begin{array}{ll}-0.709 & 0.000\end{array}$

$\begin{array}{ll}-0.689 & 0.559\end{array}$

$-0.419 \quad 0.182$

$\begin{array}{ll}-1.129 & 0.559\end{array}$

Nd II $\quad 4947.020$

4959.120

$\begin{array}{ll}-0.799 & 0.064\end{array}$

$5092.790 \quad-0.609 \quad 0.380$

5249.580

5255.510

0.200

0.976

$\begin{array}{ll}-0.669 & 0.205\end{array}$

Nd II $\quad 5293.160$

5306.460

5311.450

0.100

0.823

$\begin{array}{ll}-0.969 & 0.859\end{array}$

$\begin{array}{ll}-0.419 & 0.986\end{array}$

Nd II $\quad 5319.810$

5356.970

5485.700

$-0.139$

0.550

$\mathrm{Nd}$ II

$-0.279$

1.264

$-0.119 \quad 1.264$

$\begin{array}{ll}-1.229 & 0.559\end{array}$

Nd II $\quad 5533.820$

5548.450

$-1.269$

0.550

Sm II $\quad 4188.125$

Sm II 4424.321

Sm II $\quad 4434.320$

Sm II $\quad 4452.722$

Sm II $\quad 4467.341$

Sm II $\quad 4499.475$

Sm II $\quad 4511.830$

$-0.440$

0.543

0.484

$0.070 \quad 0.378$

$\begin{array}{ll}-0.410 & 0.277\end{array}$

$\begin{array}{ll}0.150 & 0.659\end{array}$

$0.870 \quad 0.248$
-0.820

$\begin{array}{ll}-0.820 & 0.184\end{array}$ 
4398 T. Mishenina et al.

Table A1 - continued

\begin{tabular}{lcrcr}
\hline El & $\begin{array}{c}\lambda \\
0.1 \mathrm{~nm}\end{array}$ & $\log g f$ & $\begin{array}{c}E_{\text {low }} \\
\mathrm{eV}\end{array}$ & Note \\
\hline Sm II & 4523.909 & -0.390 & 0.439 & \\
Sm II $_{\text {Sm II }}^{4536.512}$ & -1.280 & 0.104 & \\
Sm II $_{\text {Eu II }}^{4577.688}$ & -0.650 & 0.248 & \\
Eu II $_{\text {Gd }}$ & 4642.228 & -0.460 & 0.378 & \\
Gd III $_{\text {Gd }}$ & 4129.720 & 0.220 & 0.000 & \\
Gd II $_{\text {II }}^{6645.060}$ & 0.120 & 1.380 & \\
Gd II $^{4085.558}$ & -0.010 & 0.731 & \\
\hline
\end{tabular}

Table A2. Comparison of atmospheric parameters with the data of other authors.

\begin{tabular}{|c|c|c|c|c|}
\hline HD & $T_{\text {eff }}$ & $\log g$ & {$[\mathrm{Fe} / \mathrm{H}]$} & Sources \\
\hline \multirow[t]{11}{*}{6582} & 5308 & 4.41 & -0.89 & Jofré et al. (2014) \\
\hline & 5412 & 4.56 & -0.8 & Ramírez et al. (2013) \\
\hline & 5526 & 4.49 & -0.77 & Gray et al. (2003) \\
\hline & 5240 & 4.3 & -0.94 & Mishenina et al. (2011) \\
\hline & 5291 & 4.57 & -0.89 & Maldonado et al. (2012) \\
\hline & 5331 & 4.54 & -0.81 & Takeda (2007) \\
\hline & 5387 & 4.51 & -0.83 & Zhao \& Gehren (2000) \\
\hline & 5390 & 4.45 & -0.83 & Mashonkina \& Gehren (2000) \\
\hline & 5250 & 4.40 & -0.98 & Fulbright (2000) \\
\hline & 5240 & 4.20 & -0.89 & Mishenina \& Kovtyukh (2001) \\
\hline & 5322 & 4.46 & -0.82 & Gratton et al. (2003) \\
\hline Mean & $5336 \pm 87$ & $4.44 \pm 11$ & $-0.86 \pm 0.06$ & \\
\hline \multirow[t]{4}{*}{6833} & 4450 & 1.4 & -1.04 & Fulbright (2000) \\
\hline & 4400 & 1.5 & -0.85 & Mashonkina, Ryabtsev \& Frebel (2012) \\
\hline & 4450 & 1.4 & -1.04 & Molenda-Żakowicz et al. (2013) \\
\hline & 4400 & 1 & -0.89 & Mishenina \& Kovtyukh (2001) \\
\hline Mean & $4425 \pm 29$ & $1.32 \pm 0.22$ & $-0.96 \pm 0.10$ & \\
\hline \multirow[t]{15}{*}{19445} & 5820 & 3.65 & -2.28 & Roederer et al. (2014a) \\
\hline & 6055 & 4.43 & -1.83 & Ramírez et al. (2013) \\
\hline & 5920 & 4.3 & -1.98 & Gray et al. (2003) \\
\hline & 5982 & 4.38 & -2.13 & Hansen et al. (2013) \\
\hline & 5890 & 4.48 & -2.12 & Sozzetti et al. (2009) \\
\hline & 6016 & 4.43 & -1.95 & Zhao \& Gehren (2000) \\
\hline & 6020 & 4.38 & -1.95 & Mashonkina \& Gehren (2000) \\
\hline & 6047 & 4.51 & -1.96 & Gratton et al. (2000) \\
\hline & 5825 & 4.20 & -2.13 & Fulbright (2000) \\
\hline & 5890 & 4.48 & -2.12 & Molenda-Żakowicz et al. (2013) \\
\hline & 6136 & 4.43 & $7.35^{*}$ & VandenBerg et al. (2014) \\
\hline & 6000 & 4.00 & -1.89 & Mishenina \& Kovtyukh (2001) \\
\hline & 6135 & 4.46 & -2.01 & Casagrande et al. (2010) \\
\hline & 5890 & 4.50 & -2.04 & Klochkova et al. (2011) \\
\hline & 5976 & 4.44 & -2.04 & Gratton et al. (2003) \\
\hline Mean & $5973 \pm 99$ & $4.34 \pm 0.23$ & $-2.03 \pm 0.12$ & without VandenBerg et al. (2014) \\
\hline \multirow[t]{14}{*}{22879} & 5786 & 4.23 & -0.85 & Jofré et al. (2014) \\
\hline & 5970 & 4.52 & -0.81 & Bensby et al. (2014) \\
\hline & 5949 & 4.68 & -0.79 & Tsantaki et al. (2013) \\
\hline & 5910 & 4.30 & -0.83 & Ramírez et al. (2013) \\
\hline & 5884 & 4.52 & -0.82 & Adibekyan et al. (2012) \\
\hline & 5759 & 4.25 & -0.85 & Nissen \& Schuster (2011) \\
\hline & 5972 & 4.5 & -0.77 & Mishenina et al. (2011) \\
\hline & 5827 & 4.45 & -0.69 & Sozzetti et al. (2009) \\
\hline & 5774 & 4.20 & -0.86 & Nissen et al. (2000) \\
\hline & 5870 & 4.27 & -0.86 & Mashonkina \& Gehren (2000) \\
\hline & 5800 & 4.30 & -0.91 & Fulbright (2000) \\
\hline & 5857 & 4.46 & -0.83 & Sousa et al. (2008) \\
\hline & 5775 & 4.26 & -0.83 & Yan, Shi \& Zhao (2015) \\
\hline & 5800 & 4.29 & -0.84 & Sitnova et al. (2015) \\
\hline
\end{tabular}


Table A2 - continued

\begin{tabular}{|c|c|c|c|c|}
\hline HD & $T_{\text {eff }}$ & $\log g$ & {$[\mathrm{Fe} / \mathrm{H}]$} & Sources \\
\hline & 5941 & 4.41 & -0.91 & Casagrande et al. (2010) \\
\hline & 5802 & 4.37 & -0.78 & Klochkova et al. (2011) \\
\hline & 5827 & 4.44 & -0.79 & Gratton et al. (2003) \\
\hline Mean & $5853 \pm 73$ & $4.37 \pm 0.13$ & $-0.83 \pm 0.05$ & \\
\hline \multirow[t]{13}{*}{84937} & 6275 & 4.11 & -2.08 & Jofré et al. (2014) \\
\hline & 6541 & 4.23 & -1.92 & Bensby et al. (2014) \\
\hline & 6431 & 4.08 & -2.15 & Ishigaki et al. (2012) \\
\hline & 6377 & 4.15 & -2.02 & Ramírez et al. (2013) \\
\hline & 6206 & 3.89 & -2.20 & Boesgaard et al. (2011) \\
\hline & 6350 & 4.03 & -2.07 & Mashonkina \& Gehren (2000) \\
\hline & 6375 & 4.1 & -2.08 & Fulbright (2000) \\
\hline & 6431 & 4.08 & -2.15 & Lind et al. (2013) \\
\hline & 6350 & 4.09 & -2.12 & Sitnova et al. (2015) \\
\hline & 6408 & 3.93 & -2.11 & Casagrande et al. (2010) \\
\hline & 6300 & 4.00 & -2.15 & Lawler et al. (2013) \\
\hline & 6250 & 3.80 & -2.00 & Mishenina \& Kovtyukh (2001) \\
\hline & 6290 & 4.02 & -2.18 & Gratton et al. (2003) \\
\hline Mean & $6353 \pm 90$ & $4.04 \pm 0.11$ & $-2.09 \pm 0.08$ & \\
\hline \multirow[t]{13}{*}{103095} & 4827 & 4.6 & -1.34 & Jofré et al. (2014) \\
\hline & 5149 & 4.71 & -1.27 & Ramírez et al. (2013) \\
\hline & 5157 & 4.76 & -1.08 & Gray et al. (2003) \\
\hline & 5144 & 4.05 & -1.12 & Maldonado et al. (2012) \\
\hline & 5095 & 4.79 & -1.29 & Takeda (2007) \\
\hline & 5014 & 4.75 & -1.44 & Sozzetti et al. (2009) \\
\hline & 5110 & 4.67 & -1.35 & Zhao \& Gehren (2000) \\
\hline & 5110 & 4.66 & -1.35 & Mashonkina \& Gehren (2000) \\
\hline & 5152 & 4.77 & -1.17 & Gratton et al. (2000) \\
\hline & 4950 & 4.50 & -1.46 & Fulbright (2000) \\
\hline & 5000 & 4.40 & -1.39 & Mishenina \& Kovtyukh (2001) \\
\hline & 5130 & 4.66 & -1.26 & Sitnova et al. (2015) \\
\hline & 5025 & 4.63 & -1.28 & Gratton et al. (2003) \\
\hline Mean & $5066 \pm 99$ & $4.61 \pm 0.20$ & $-1.29 \pm 0.12$ & \\
\hline \multirow[t]{2}{*}{170153} & 6173 & 4.22 & -0.58 & Ramírez et al. (2013) \\
\hline & 6034 & 4.28 & -0.65 & Chen et al. (2000) \\
\hline Mean & $6104 \pm 98$ & $4.25 \pm 0.04$ & $-0.62 \pm 0.05$ & \\
\hline \multirow{4}{*}{216143} & 4525 & 1 & -2.25 & Fulbright (2000) \\
\hline & 4525 & 0.80 & -2.18 & Burris et al. (2000) \\
\hline & 4525 & 1.00 & -2.25 & Molenda-Żakowicz et al. (2013) \\
\hline & 4529 & 1.30 & -2.1 & Ishigaki et al. (2014) \\
\hline Mean & $4526 \pm 2$ & $1.03 \pm 0.21$ & $-2.20 \pm 0.07$ & \\
\hline \multirow[t]{7}{*}{221170} & 4500 & 0.9 & -2.19 & Fulbright (2000) \\
\hline & 4425 & 1.00 & -2.15 & Burris et al. (2000) \\
\hline & 4510 & 1.00 & -2.16 & Mashonkina et al. (2012) \\
\hline & 4444 & 0.92 & -2.12 & Molenda-Żakowicz et al. (2013) \\
\hline & 4510 & 1.00 & -2.09 & Ivans et al. (2006) \\
\hline & 4475 & 1.00 & -2.09 & Yushchenko et al. (2005) \\
\hline & 4500 & 1.00 & -2.05 & Mishenina \& Kovtyukh (2001) \\
\hline Mean & $4481 \pm 34$ & $0.97 \pm 0.04$ & $-2.12 \pm 0.05$ & \\
\hline \multirow[t]{11}{*}{224930} & 5510 & 4.46 & -0.76 & Ramírez et al. (2013) \\
\hline & 5502 & 4.27 & -0.64 & Gray et al. (2003) \\
\hline & 5300 & 4.10 & -0.91 & Mishenina et al. (2011) \\
\hline & 5491 & 4.75 & -0.72 & Maldonado et al. (2012) \\
\hline & 5680 & 4.86 & -0.52 & Takeda (2007) \\
\hline & 5275 & 4.10 & -1.00 & Fulbright (2000) \\
\hline & 5357 & 4.32 & -0.9 & Molenda-Żakowicz et al. (2013) \\
\hline & 5480 & 4.45 & -0.66 & Yan et al. (2015) \\
\hline & 5300 & 4.10 & -0.85 & Mishenina \& Kovtyukh (2001) \\
\hline & 5470 & 4.20 & -0.71 & Stonkute et al. (2012) \\
\hline & 5357 & 4.32 & -0.87 & Gratton et al. (2003) \\
\hline Mean & $5429 \pm 122$ & $4.36 \pm 0.26$ & $-0.78 \pm 0.14$ & \\
\hline
\end{tabular}

This paper has been typeset from a $\mathrm{T}_{\mathrm{E}} \mathrm{X} / \mathrm{L} \mathrm{T}_{\mathrm{E}} \mathrm{X}$ file prepared by the author. 